\title{
Generalized Euler-Lagrange Equations for Fuzzy Fractional Variational Problems under gH-Atangana-Baleanu Differentiability
}

\author{
Jianke Zhang (iD, Gaofeng Wang, Xiaobin Zhi, and Chang Zhou \\ Department of Mathematics, School of Science, Xian University of Posts and Telecommunications, Xian, China \\ Correspondence should be addressed to Jianke Zhang; jiankezh@163.com
}

Received 14 September 2017; Accepted 1 January 2018; Published 19 March 2018

Academic Editor: Talat Nazir

Copyright (c) 2018 Jianke Zhang et al. This is an open access article distributed under the Creative Commons Attribution License, which permits unrestricted use, distribution, and reproduction in any medium, provided the original work is properly cited.

We study in this paper the Atangana-Baleanu fractional derivative of fuzzy functions based on the generalized Hukuhara difference. Under the condition of gH-Atangana-Baleanu fractional differentiability, we prove the generalized necessary and sufficient optimality conditions for problems of the fuzzy fractional calculus of variations with a Lagrange function. The new kernel of gH-Atangana-Baleanu fractional derivative has no singularity and no locality, which was not precisely illustrated in the previous definitions.

\section{Introduction}

Fractional calculus, as an extension of ordinary calculus, has about a 300-year-old history, which [1] plays an important role in dealing with many natural dynamical processes. The fractional variational problem is an important dynamical system. In recent years, under different differentiability, several researchers [2-9] studied the fractional Euler-Lagrange equations for general fractional variational problems.

It is well known that the Riemann-Liouville (RL) and Caputo derivatives have some drawbacks. The RL derivative of a constant is not zero, and it demands initial conditions of noninteger order which are not physically determined. On the other hand, the Caputo derivative requires higher conditions of regularity for differentiability, which is specified only for differentiable functions. In 2015, a new fractional derivative [10] with a nonsingular kernel was proposed. Losada and Nieto studied several properties of the CaputoFabrizio (CF) fractional derivative [11] and other researchers tried to study the different equations (see [12] and the references therein). Under the CF differentiability, Zhang et al. [9] proposed the necessary and sufficient optimality conditions for problems of the fractional calculus of variations with a Lagrange function. However, Sheikh et al. [13, 14] pointed out that the kernel of the $\mathrm{CF}$ fractional derivative was nonsingular but was still nonlocal. Some researchers $[15,16]$ also concluded that the CF fractional derivative was not a derivative with a fractional order instead of a filter with a fractional parameter.

To overcome these drawbacks, Yang et al. [17] proposed a new fractional derivative involving the normalized sinc function without singular kernel. Based on the generalized Mittag-Leffler function, a new operator with fractional order [18] was introduced by Atangana and Baleanu in 2016. The kernel of the Atangana-Baleanu $(A B)$ fractional derivative is nonlocal and nonsingular, which have all the benefits of the $\mathrm{CF}$ derivative. In [19], the $\mathrm{AB}$ and $\mathrm{CF}$ derivatives were used in the Allen-Cahn reaction-diffusion model, and the modified models were solved numerically and numerical simulations were presented for different values of alpha. Abdeljawad and Baleanu [20] studied some properties of the AB fractional derivative.

However, the majority of real-world optimization problems are often involved in data uncertainty or imprecision owing to measurement errors or some unexpected things. Fuzziness is a kind of very common uncertainty in the problems of the real world. Farhadinia [21] was the first one who introduced the concept of fuzzy variational problem and studied the optimality conditions for this problem. Fard and Salehi [22] and Soolaki et al. [23] also established the 
necessary optimality conditions for fuzzy fractional variational problems using the concept of Caputo and combined Caputo differentiability based on Hukuhara difference of fuzzy functions.

In this paper, we extend the concept of Atangana-Baleanu fractional derivative to fuzzy functions based on the generalized Hukuhara difference. Under the condition of $\mathrm{gH}$ Atangana-Baleanu fractional differentiability, we prove the generalized necessary and sufficient optimality conditions for problems of the fuzzy fractional calculus of variations with a Lagrange function. In our results, the fuzzy admissible curve $\tilde{x}(t)=[\underline{x}(t, \alpha), \bar{x}(t, \alpha)]$ is generalized Hukuhara differentiable, and the two real-valued functions $\underline{x}(t, \alpha)$ and $\bar{x}(t, \alpha)$ are differentiable, which generalized the results of [21-23]. The new kernel of gH-Atangana-Baleanu fractional derivative has no singularity and no locality, which was not precisely illustrated in the previous definitions.

The paper is organized as follows. Section 2 presents some preliminaries needed in the sequel. The notion of the Atangana-Baleanu fuzzy fractional derivative is defined in Section 3. In Section 4, the generalized fuzzy fractional EulerLagrange conditions for the fuzzy fractional variational problems with natural boundary conditions are obtained. In Section 5 , the weakly sufficient optimality conditions for fuzzy fractional variational problems are proposed. Finally, we give a conclusion in Section 6.

\section{Preliminaries}

In this section, we recall some definitions and basic concepts which will be used in this paper. Let $\mathbf{R}^{n}$ be an $n$-dimensional Euclidean space and let $\mathbf{R}_{+}^{n}$ be its nonnegative orthant. A fuzzy set on $\mathbf{R}^{n}$ is a mapping $\tilde{u}: \mathbf{R}^{n} \rightarrow[0,1]$. For each fuzzy set $\tilde{u}$, we denote its $\alpha$-level set as $[\tilde{u}]^{\alpha}=\left\{x \in \mathbf{R}^{n} \mid \tilde{u}(x) \geq \alpha\right\}$ for any $\alpha \in(0,1]$ and supp $u=\left\{x \in \mathbf{R}^{n}: \widetilde{u}(x)>0\right\}$ (called support of $\tilde{u})$; the closure of supp $u$ in $\mathbf{R}^{n}$ is written as $[\widetilde{u}]^{0}$.

Definition 1. A compact and convex fuzzy set $\tilde{u}$ on $\mathbf{R}^{n}$ is a fuzzy set with the following properties:

(1) $\tilde{u}$ is normal; that is, there exists $x_{0} \in \mathbf{R}^{n}$ such that $\widetilde{u}\left(x_{0}\right)=1$.

(2) $\tilde{u}$ is an upper semicontinuous function.

(3) $\tilde{u}(\lambda x+(1-\lambda) y) \geq \min \{\tilde{u}(x), \tilde{u}(y)\}, x, y \in \mathbf{R}^{n}, \lambda \in$ $[0,1]$.

(4) supp $\tilde{u}=\left\{x \in \mathbf{R}^{n} \mid \tilde{u} \geq 0\right\}$ is the support of $\tilde{u}$ and its closure $\mathrm{cl}(\operatorname{supp} \widetilde{u})$ is compact.

It is well known that the $\alpha$-cut set of a fuzzy number is a closed and bounded interval $\left[\underline{u}^{\alpha}(x), \bar{u}^{\alpha}(x)\right]$ for any $\alpha \in[0,1]$.

Definition 2. The left-hand endpoint $\underline{u}^{\alpha}(x)$ and the righthand endpoint $\bar{u}^{\alpha}(x)$ of a fuzzy number $\widetilde{u}(x)$ satisfy the following conditions:

(1) $\underline{u}^{\alpha}(x)$ is a monotonically increasing left continuous function.

(2) $\bar{u}^{\alpha}(x)$ is a monotonically decreasing left continuous function.
(3) $\underline{u}^{\alpha}(x) \leq \bar{u}^{\alpha}(x), 0 \leq \alpha \leq 1$.

For the two fuzzy numbers $[\widetilde{u}]^{\alpha}=\left[\underline{u}^{\alpha}, \bar{u}^{\alpha}\right]$ and $[\widetilde{v}]^{\alpha}=$ $\left[\underline{v}^{\alpha}, \bar{v}^{\alpha}\right]$, the sum $[\widetilde{u}]^{\alpha}+[\widetilde{v}]^{\alpha}$ and the product $k \cdot[\widetilde{u}]^{\alpha}$ for $k \in \mathbf{R}$ and all $0 \leq \alpha \leq 1$ are defined as

$$
\begin{aligned}
& {[\tilde{u}+\widetilde{v}]^{\alpha}=\left[\underline{u}^{\alpha}+\underline{v}^{\alpha}, \bar{u}^{\alpha}+\bar{v}^{\alpha}\right] ;} \\
& {[\widetilde{u}+\widetilde{v}]^{\alpha}=\left[\underline{u}^{\alpha}-\bar{v}^{\alpha}, \bar{u}^{\alpha}-\underline{v}^{\alpha}\right] .}
\end{aligned}
$$

$k \leq 0$

$[k \tilde{u}]^{\alpha}=\left[k \underline{u}^{\alpha}, k \bar{u}^{\alpha}\right]$ if $k \geq 0$ and $[k \tilde{u}]^{\alpha}=\left[k \bar{u}^{\alpha}, k \underline{u}^{\alpha}\right]$ if

The product of the two fuzzy numbers $[\tilde{u}]^{\alpha}=\left[\underline{u}^{\alpha}, \bar{u}^{\alpha}\right]$ and $[\widetilde{v}]^{\alpha}=\left[\underline{v}^{\alpha}, \bar{v}^{\alpha}\right]$ is defined as

$$
\begin{gathered}
{[\tilde{u} \odot \widetilde{v}]^{\alpha}=\left[\min \left\{\underline{u}^{\alpha} \underline{v}^{\alpha}, \underline{u}^{\alpha} \bar{v}^{\alpha}, \bar{u}^{\alpha} \underline{v}^{\alpha}, \bar{u}^{\alpha} \bar{v}^{\alpha}\right\},\right.} \\
\left.\max \left\{\underline{u}^{\alpha} \underline{v}^{\alpha}, \underline{u}^{\alpha} \bar{v}^{\alpha}, \bar{u}^{\alpha} \underline{v}^{\alpha}, \bar{u}^{\alpha} \bar{v}^{\alpha}\right\}\right] .
\end{gathered}
$$

Let us denote the class of fuzzy numbers by $\mathscr{R}_{f}$. For $\tilde{u}, \tilde{v} \in$ $\mathscr{R}_{f}$, the Hausdorff distance $d: \mathscr{R}_{f} \times \mathscr{R}_{f} \rightarrow \mathbf{R}_{+} \cup\{0\}$ is defined as

$$
d(\widetilde{u}, \widetilde{v})=\sup _{\alpha \in[0,1]} \max \left\{\left|\underline{u}^{\alpha}-\underline{v}^{\alpha}\right|,\left|\bar{u}^{\alpha}-\bar{v}^{\alpha}\right|\right\} .
$$

It can be seen that the distance $d$ is a metric in $\mathscr{R}_{f}$, and it has the following properties:

(1) $d(\widetilde{u}+\widetilde{w}, \widetilde{v}+\widetilde{w})=d(\widetilde{u}, \widetilde{v}), \forall \widetilde{u}, \widetilde{w}, \widetilde{v} \in \mathscr{R}_{f}$,

(2) $d(k \tilde{u}, k \widetilde{v})=|k| d(\tilde{u}, \widetilde{v}), \forall \tilde{u}, \widetilde{v} \in \mathscr{R}_{f}, k \in \mathbf{R}$,

(3) $d(\widetilde{u}+\widetilde{v}, \widetilde{w}+\widetilde{m}) \leq d(\widetilde{u}, \widetilde{v})+d(\widetilde{w}, \widetilde{m}), \forall \widetilde{u}, \widetilde{w}, \widetilde{m}, \widetilde{v} \in \mathscr{R}_{f}$,

(4) $\left(d, \mathscr{R}_{f}\right)$ is a complete metric space.

Definition 3 (see [24]). The generalized Hukuhara difference of two fuzzy numbers $\tilde{x} \in \mathscr{R}_{f}$ and $\tilde{y} \in \mathscr{R}_{f}$ (gH-difference for short) is defined as follows:

$$
\begin{aligned}
\tilde{x} \ominus_{\mathrm{gH}} \tilde{y} & =\widetilde{z} \Longleftrightarrow \\
\tilde{x} & =\tilde{y}+\widetilde{z} \\
\text { or } \tilde{y} & =\tilde{x}+(-1) \widetilde{z} .
\end{aligned}
$$

If $\widetilde{z}=\tilde{x} \ominus_{\mathrm{gH}} \tilde{y}$ exists as a fuzzy number, then its level cuts $\left[\underline{z}^{\alpha}, \bar{z}^{\alpha}\right]$ are given by

$$
\begin{aligned}
& \underline{z}^{\alpha}=\min \left\{\underline{x}^{\alpha}-\underline{y}^{\alpha}, \bar{x}^{\alpha}-\bar{y}^{\alpha}\right\}, \\
& \bar{z}^{\alpha}=\max \left\{\underline{x}^{\alpha}-\underline{y}^{\alpha}, \bar{x}^{\alpha}-\bar{y}^{\alpha}\right\}
\end{aligned}
$$

for all $\alpha \in[0,1]$.

Definition 4 (see [21]). Let $\tilde{a} \in \mathscr{R}_{f}$ and $\tilde{b} \in \mathscr{R}_{f}$. One writes $\tilde{a} \preceq \tilde{b}$ if $\underline{a}^{\alpha} \leq \underline{b}^{\alpha}$ and $\bar{a}^{\alpha} \leq \bar{b}^{\alpha}$ for all $\alpha \in[0,1]$. One also writes $\tilde{a} \prec \tilde{b}$ if $\tilde{a} \preceq \tilde{b}$, and there exists an $\alpha^{*} \in[0,1]$ so that $\underline{a}^{\alpha^{*}}<\underline{b}^{\alpha^{*}}$ and $\bar{a}^{\alpha^{*}}<\bar{b}^{\alpha^{*}}$. Moreover, $\widetilde{a} \approx \widetilde{b}$ if $\widetilde{a} \preceq \widetilde{b}$ and $\widetilde{a} \succeq \tilde{b}$; that is, $[a]^{\alpha}=[b]^{\alpha}$ for all $\alpha \in[0,1]$. 
We say that $\tilde{a}, \widetilde{b} \in \mathscr{R}_{f}$ are comparable if either $\widetilde{a} \leq \widetilde{b}$ or $\tilde{a} \geq \widetilde{b}$ and noncomparable otherwise.

A function $\widetilde{F}: K \rightarrow \mathscr{R}_{f}, K \subseteq \mathbf{R}^{n}$ is said to be a fuzzy function. For each $\alpha \in[0,1]$, we associate with $\widetilde{F}$ the family of interval-valued functions given by $\widetilde{F}^{\alpha}(t)=[f(t, \alpha), \bar{f}(t, \alpha)]$. Here, the endpoint functions $f(t, \alpha), \bar{f}(t, \alpha): K \rightarrow \mathbf{R}$ are called lower and upper functions of $\widetilde{F}$, respectively.

Definition 5 (see [24]). Let $t \in(a, b)$ and $h$ be such that $t+h \in$ $(a, b)$; then, the generalized Hukuhara derivative of a fuzzyvalued function $\widetilde{x}(t):(a, b) \rightarrow \mathscr{R}_{f}$ at $t$ is defined as

$$
D_{\mathrm{gH}} \tilde{x}(t)=\lim _{h \rightarrow 0} \frac{\tilde{x}(t+h) \ominus_{\mathrm{gH}} \tilde{x}(t)}{h} .
$$

If $D_{\mathrm{gH}} \tilde{x}(t) \in \mathscr{R}_{f}$ exists, we say that $\tilde{x}$ is generalized Hukuhara differentiable (gH-differentiable for short) at $t$.

Definition 6 (see [21]). One says that the fuzzy function $\tilde{x}$ : $[a, b] \rightarrow \mathscr{R}_{f}$ with $\alpha$-level set $[x(t)]^{\alpha}=[\underline{x}(t, \alpha), \bar{x}(t, \alpha)]$ is continuous at $t \in[a, b]$, if crisp functions $\underline{x}(t, \alpha)$ and $\bar{x}(t, \alpha)$ are continuous functions at $t \in[a, b]$ for all $\alpha \in[0,1]$.

If the fuzzy function $\tilde{x}:[a, b] \rightarrow \mathscr{R}_{f}$ is continuous in the metric $d(\cdot, \cdot)$, then its definite integral exists. Furthermore,

$$
\begin{aligned}
& \left(\underline{\left.\frac{\int_{a}^{b} \tilde{x}(t)}{} d t\right)^{\alpha}}=\int_{a}^{b} \underline{x}(t, \alpha) d t,\right. \\
& \left(\overline{\left.\int_{a}^{b} \tilde{x}(t) d t\right)^{\alpha}=\int_{a}^{b} \bar{x}(t, \alpha) d t .}\right.
\end{aligned}
$$

Theorem 7 (see [25]). Let $\tilde{x}:[a, b] \rightarrow \mathscr{R}_{f}$ be a fuzzy function. If $\tilde{x}$ is $g H$-differentiable at $t_{0} \in[a, b]$, then for each $\alpha \in[0,1]$, one of the following cases holds:

(a) $\underline{x}^{\alpha}$ and $\bar{x}^{\alpha}$ are differentiable at $t_{0}$ and

$$
\begin{aligned}
& {\left[D_{g H} \tilde{x}\left(t_{0}\right)\right]^{\alpha}=\left[\min \left\{\underline{x}^{\prime}\left(t_{0}, \alpha\right), \bar{x}^{\prime}\left(t_{0}, \alpha\right)\right\},\right.} \\
& \left.\quad \max \left\{\underline{x}^{\prime}\left(t_{0}, \alpha\right), \bar{x}^{\prime}\left(t_{0}, \alpha\right)\right\}\right] ;
\end{aligned}
$$

(b) $(\underline{x})_{-}^{\prime}\left(t_{0}, \alpha\right),(\underline{x})_{+}^{\prime}\left(t_{0}, \alpha\right),(\bar{x})_{-}^{\prime}\left(t_{0}, \alpha\right)$ and $(\bar{x})_{+}^{\prime}\left(t_{0}, \alpha\right)$ exist and these satisfy $(\underline{x})_{-}^{\prime}\left(t_{0}, \alpha\right)=(\bar{x})_{+}^{\prime}\left(t_{0}, \alpha\right)$ and $(\underline{x})_{+}^{\prime}\left(t_{0}, \alpha\right)=(\bar{x})_{-}^{\prime}\left(t_{0}, \alpha\right)$. Moreover,

$$
\begin{aligned}
& {\left[D_{g H} \tilde{x}\left(t_{0}\right)\right]^{\alpha}=\left[\min \left\{(\underline{x})_{-}^{\prime}\left(t_{0}, \alpha\right),(\bar{x})_{-}^{\prime}\left(t_{0}, \alpha\right)\right\},\right.} \\
& \left.\quad \max \left\{(\underline{x})_{-}^{\prime}\left(t_{0}, \alpha\right),(\bar{x})_{-}^{\prime}\left(t_{0}, \alpha\right)\right\}\right] \\
& \quad=\left[\operatorname { m i n } \left\{\left(\underline{x}_{+}^{\prime}\left(t_{0}, \alpha\right),(\bar{x})_{+}^{\prime}\left(t_{0}, \alpha\right)\right\},\right.\right. \\
& \left.\quad \max \left\{(\underline{x})_{+}^{\prime}\left(t_{0}, \alpha\right),(\bar{x})_{+}^{\prime}\left(t_{0}, \alpha\right)\right\}\right] .
\end{aligned}
$$

Proposition 8 (see [25]). Let $\widetilde{F}_{\alpha}(x)=[f(x, \alpha), \bar{f}(x, \alpha)]$ : $K \rightarrow \mathscr{R}_{f}$ be a fuzzy function. If $\widetilde{F}$ is $g \bar{H}$-differentiable at $x_{0} \in K$, then for each $\alpha \in[0,1]$, the real-valued function $f(x, \alpha)+\bar{f}(x, \alpha): K \rightarrow \mathbf{R}$ is differentiable at $x_{0}$. Moreover,

$$
\frac{\partial F_{\alpha}}{\partial x_{i}}\left(x_{0}\right)+\frac{\partial \overline{F_{\alpha}}}{\partial x_{i}}\left(x_{0}\right)=\frac{\partial(\underline{f}(x, \alpha)+\bar{f}(x, \alpha))}{\partial x_{i}}\left(x_{0}\right) .
$$

Remark 9. Chalco-Cano et al. [25] have pointed that the concept of generalized Hukuhara differentiable fuzzy functions is more general than level-wise differentiability and Hukuhara differentiability. For a gH-differentiable fuzzy function $\widetilde{F}_{\alpha}(x)=[f(x, \alpha), \bar{f}(x, \alpha)]: K \rightarrow \mathscr{R}_{f}$, the endpoint functions $f(x, \alpha)$ and $\bar{f}(x, \alpha)$ are not necessarily differentiable.

Definition 10 (see [23]). Let $T$ be an open subset of $\mathbf{R}$ and $\widetilde{F}$ : $T \rightarrow \mathscr{R}_{f}$ and $x_{0} \in T$. One says that $\widetilde{F}\left(x_{0}\right)$ is a local minimum (maximum) of $\widetilde{F}(\cdot)$ if there exists some $\epsilon>0$ such that $\widetilde{F}\left(x_{0}\right) \preceq(\succeq) \widetilde{F}(x)$ when $x \in N_{\epsilon}\left(x_{0}\right)=\left\{x \in T \mid\left\|x-x_{0}\right\| \leq \epsilon\right\}$.

Theorem 11 (see [26]). Let $f$ be a real-valued function differentiable on the open interval I. If $f$ has a local extremum at $x \in I$, then $(d / d x) f(x)=0$.

Theorem 12 (see [23]). Let $\widetilde{F}:[a, b] \rightarrow \mathscr{R}_{f}$ be a fuzzy function. If the local minimum of $\widetilde{F}$ is attended in the point $x^{*} \in[a, b]$, then the local minimum of real-valued endpoint functions $f(x, \alpha)$ and $\bar{f}(x, \alpha)$ is attended in the point $x^{*}$ for all $\alpha \in[0,1]$. So, one has

$$
\frac{d \underline{f}(x, \alpha)}{d x}\left(x^{*}\right)=\frac{d \bar{f}(x, \alpha)}{d x}\left(x^{*}\right)=0 .
$$

\section{Fuzzy Atangana-Baleanu Fractional Derivative}

We recall some basic concepts with regard to the $\mathrm{RL}$ fractional calculus, Caputo fractional derivative, and the AtanganaBaleanu fractional derivative [18]. Given a function $x(t)$ : $[a, b] \rightarrow \mathbf{R}$, the RL fractional calculus of $x$ of order $\gamma \in(0,1)$ is defined as follows:

(1) The left RL fractional integral of order $\gamma>0$ starting from $a$ is defined by

$$
{ }^{\mathrm{RL}} I_{a+}^{\gamma} x(t)=\frac{1}{\Gamma(\gamma)} \int_{a}^{t}(t-\tau)^{\gamma-1} x(\tau) d \tau .
$$

(2) The right RL fractional integral of order $\gamma>0$ ending at $b>a$ is defined by

$$
{ }^{\mathrm{RL}} I_{b_{-}}^{\gamma} x(t)=\frac{1}{\Gamma(\gamma)} \int_{t}^{b}(\tau-t)^{\gamma-1} x(\tau) d \tau .
$$

(3) The left RL fractional derivative of order $0<\gamma<1$ starting at $a$ is defined by

$$
{ }^{\mathrm{RL}} D_{a+}^{\gamma} x(t)=\frac{d}{d t}\left[{ }^{\mathrm{RL}} I_{a+}^{1-\gamma} x(t)\right] .
$$


(4) The right RL fractional derivative of order $0<\gamma<1$ ending at $b$ is defined by

$$
{ }^{\mathrm{RL}} D_{b-}^{\gamma} x(t)=-\frac{d}{d t}\left[{ }^{\mathrm{RL}} I_{b-}^{1-\gamma} x(t)\right] .
$$

(5) The left Caputo fractional derivative of order $0<\gamma<$ 1 starting at $a$ is defined by

$$
\begin{aligned}
& { }^{C} D_{a+}^{\gamma} x(t) \\
& \quad=\frac{1}{\Gamma(1-\gamma)} \frac{d}{d t} \int_{a}^{t} \frac{1}{(t-\tau)^{\gamma}}[x(\tau)-x(a)] d \tau .
\end{aligned}
$$

If $x$ is of class $C^{1}$, then

$$
{ }^{C} D_{a+}^{\gamma} x(t)=\frac{1}{\Gamma(1-\gamma)} \int_{a}^{t} \frac{1}{(t-\tau)^{\gamma}} x^{\prime}(\tau) d \tau .
$$

The new Caputo-Fabrizio (CF) fractional derivative [12, 15] can be obtained by changing the kernel $(t-\tau)^{-\gamma}$ by the function $\exp (-\gamma(t-\tau) /(1-\gamma))$ and $1 / \Gamma(1-\gamma)$ with $M(\gamma) /(1-$ $\gamma)$. That is,

$$
{ }^{\mathrm{CF}} D_{a+}^{\gamma} x(t)=\frac{M(\gamma)}{1-\gamma} \int_{a}^{t} \exp \left(-\frac{\gamma(t-\tau)}{1-\gamma}\right) x^{\prime}(\tau) d \tau,
$$

where $M(\gamma)$ is a normalization function such that $M(0)=$ $M(1)=1$. It is clear that if $x$ is a constant function, then ${ }^{\mathrm{CF}} D^{\gamma} x(t)=0$ as in the usual Caputo derivative, but contrary to the usual Caputo derivative, the kernel does not have singularity for $t=\tau$.

However, Atangana and Koca [15] pointed out that the integral associate is not a fractional operator but the average of the function and its integral. Some researchers also concluded that the operator was not a derivative with a fractional order instead of a filter with a fractional parameter. The fractional parameter can then be viewed as a filter regulator. To solve the above drawbacks, Atangana and Baleanu introduced a new operator with a fractional order based upon the generalized Mittag-Leffler function [18] as follows.

Definition 13 (see [18]). Let $x \in H^{1}(a, b), b>a, \gamma \in$ $(0,1)$; then, the definition of the Atangana-Baleanu fractional derivative in the sense of left Caputo is described as

$$
{ }^{\mathrm{ABC}} D_{a^{+}}^{\gamma} x(t)=\frac{B(\gamma)}{1-\gamma} \int_{a}^{t} E_{\gamma}\left(-\frac{\gamma(t-\tau)^{\gamma}}{1-\gamma}\right) x^{\prime}(\tau) d \tau .
$$

And in the left RL sense, it is described by

$$
\begin{aligned}
{ }^{\mathrm{ABR}} D_{a+}^{\gamma} x(t) \\
=\frac{B(\gamma)}{1-\gamma} \frac{d}{d t} \int_{a}^{t} E_{\gamma}\left(-\frac{\gamma(t-\tau)^{\gamma}}{1-\gamma}\right) x(\tau) d \tau .
\end{aligned}
$$

The associated fractional integral is described by

$$
{ }^{\mathrm{AB}} I_{a+}^{\gamma} x(t)=\frac{1-\gamma}{B(\gamma)} x(t)+\frac{\gamma}{B(\gamma)}\left[{ }^{\mathrm{RL}} I_{a+}^{\gamma} x(t)\right]
$$

where $B(\gamma)$ stands for normalization function such that $B(0)=B(1)=1$ and

$$
E_{\gamma}(Z)=\sum_{k=0}^{\infty} \frac{z^{k}}{\Gamma(\gamma k+1)}, \quad \operatorname{Re}(\gamma)>0, z \in C
$$

is the Mittag-Leffler function of one parameter.

Definition 14 (see [20]). The right Atangana-Baleanu fractional derivative with Mittag-Leffler kernel in the RL sense of order $\gamma \in(0,1)$ is defined by

$$
\begin{aligned}
{ }^{\mathrm{ABR}} & D_{b-}^{\gamma} x(t) \\
& =-\frac{B(\gamma)}{1-\gamma} \frac{d}{d t} \int_{t}^{b} E_{\gamma}\left(-\frac{\gamma(\tau-t)^{\gamma}}{1-\gamma}\right) x(\tau) d \tau .
\end{aligned}
$$

Definition 15 (see [20]). The right Atangana-Baleanu fractional integral with Mittag-Leffler kernel in the RL sense of order $\gamma \in(0,1)$ is defined by

$$
{ }^{\mathrm{AB}} I_{b-}^{\gamma} x(t)=\frac{1-\gamma}{B(\gamma)} x(t)+\frac{\gamma}{B(\gamma)}{ }^{\mathrm{RL}} I_{b-}^{\gamma} x(t) .
$$

Definition 16 (see [20]). The right Atangana-Baleanu fractional derivative with Mittag-Leffler kernel in the Caputo sense of order $\gamma \in(0,1)$ is defined by

$$
{ }^{\mathrm{ABC}} D_{b-}^{\gamma} x(t)=-\frac{B(\gamma)}{1-\gamma} \int_{t}^{b} E_{\gamma}\left(-\frac{\gamma(\tau-t)^{\gamma}}{1-\gamma}\right) x^{\prime}(\tau) d \tau .
$$

Proposition 17 (see [20]). The right Atangana-Baleanu fractional derivatives with Mittag-Leffler kernel in the RL sense and in the Caputo sense are related by the identity

$$
\begin{aligned}
{ }^{\mathrm{ABC}} D_{b-}^{\gamma} x(t)= & { }^{\mathrm{ABR}} D_{b-}^{\gamma} x(t) \\
& -\frac{B(\gamma)}{1-\gamma} x(b) E_{\gamma}\left(-\frac{\gamma(b-t)^{\gamma}}{1-\gamma}\right) .
\end{aligned}
$$

Proposition 18 (see [20] (integration by parts for the Atangana-Baleanu fractional derivative in the Caputo sense)). Let $x$ be a continuous function and $y$ be of class $C^{1}$. Then,

$$
\begin{aligned}
& \int_{0}^{b} x(t){ }^{\mathrm{ABC}} D_{a+}^{\gamma} y(t) d t \\
& =\int_{0}^{b} y(t){ }^{\mathrm{ABR}} D_{b-}^{\gamma} x(t) d t \\
& \quad+\left.\frac{B(\gamma)}{1-\gamma}\left[y(t) \mathbf{E}_{\gamma, 1,-\gamma /(1-\gamma), b-}^{1} x(t)\right]\right|_{t=0} ^{t=b}, \\
& \int_{0}^{b} x(t){ }^{\mathrm{ABC}} D_{b-}^{\gamma} y(t) d t \\
& =\int_{0}^{b} y(t){ }^{\mathrm{ABR}} D_{0+}^{\gamma} x(t) d t \\
& \quad-\left.\frac{B(\gamma)}{1-\gamma}\left[y(t) \mathbf{E}_{\gamma, 1,-\gamma /(1-\gamma), 0+}^{1} x(t)\right]\right|_{t=0} ^{t=b},
\end{aligned}
$$


where the left generalized fractional integral operator is defined by

$$
\mathbf{E}_{\gamma, \mu, \omega, a+}^{\alpha} x(t)=\int_{a}^{t}(t-\tau)^{\mu-1} E_{\gamma, \mu}^{\alpha}\left[\omega(t-\tau)^{\gamma}\right] x(\tau) d \tau,
$$

$$
t>a,
$$

and the right generalized fractional integral operator is defined by

$$
\begin{array}{r}
\mathbf{E}_{\gamma, \mu, \omega, b-}^{\alpha} x(t)=\int_{t}^{b}(\tau-t)^{\mu-1} E_{\gamma, \mu}^{\alpha}\left[\omega(\tau-t)^{\gamma}\right] x(\tau) d \tau, \\
t<b,
\end{array}
$$

where $E_{\gamma, \mu}^{\alpha}(z)=\sum_{k=0}^{\infty}\left((\alpha)_{k} z^{k} / \Gamma(\gamma k+\mu) k !\right)$ is the generalized Mittag-Leffler function which is defined for complex $\gamma, \mu, \alpha,(\operatorname{Re}(\gamma)>0)$.

The fuzzy gH-fractional Caputo derivative of a fuzzyvalued function was introduced in [27]. Following [27], we denote the space of all continuous fuzzy-valued functions on $[a, b] \subset \mathbf{R}$ by $C\left([a, b], \mathscr{R}_{f}\right)$, the class of fuzzy functions with continuous first derivatives on $[a, b] \subset \mathbf{R}$ by $C^{1}\left([a, b], \mathscr{R}_{f}\right)$, and the space of all Lebesgue integrable fuzzy-valued functions on the bounded interval $[a, b]$ by $L\left([a, b], \mathscr{R}_{f}\right)$.

We introduce the fuzzy gH-Atangana-Baleanu fractional derivative as follows.

Definition 19. Let $D_{\mathrm{gH}} \widetilde{x}(t) \in C\left([a, b], \mathscr{R}_{f}\right) \cap L\left([a, b], \mathscr{R}_{f}\right)$. The fuzzy gH-Atangana-Baleanu fractional differentiable fuzzy-valued function in the Caputo sense of $\widetilde{x}(t)\left([\mathrm{gH}]_{\gamma}^{\mathrm{ABC}}\right.$ differentiable for short) is defined as follows:

$$
\begin{aligned}
& \underset{\mathrm{gH}}{\mathrm{ABC}} D_{a+}^{\gamma} \tilde{x}(t) \\
& \quad=\frac{B(\gamma)}{1-\gamma} \int_{a}^{t} E_{\gamma}\left(-\frac{\gamma(t-\tau)^{\gamma}}{1-\gamma}\right)\left(D_{\mathrm{gH}} \tilde{x}\right)(\tau) d \tau,
\end{aligned}
$$

where $0<\gamma \leq 1, t>a$.

Theorem 20. Let $D_{g H} \widetilde{x}(t) \in C\left([a, b], \mathscr{R}_{f}\right) \cap L\left([a, b], \mathscr{R}_{f}\right)$ be such that $[x(t)]^{\alpha}=[\underline{x}(t, \alpha), \bar{x}(t, \alpha)]$ for $t \in[a, b]$ and $0 \leq \alpha \leq$ 1. If the real-valued functions $\underline{x}(t, \alpha)$ and $\bar{x}(t, \alpha)$ are AtanganaBaleanu-differentiable in the Caputo sense at $t \in[a, b]$, then the fuzzy function $\widetilde{x}(t)$ is $[g H]_{\gamma}^{\mathrm{ABC}}$-differentiable at $t \in[a, b]$ and

$$
\begin{aligned}
& {\left[{ }_{g H}{ }_{g B} D_{a+}^{\gamma} \tilde{x}(t)\right]^{\alpha}} \\
& \quad=\left[\min \left\{{ }^{\mathrm{ABC}} D_{a+}^{\gamma} \underline{x}(t, \alpha),{ }^{\mathrm{ABC}} D_{a+}^{\gamma} \bar{x}(t, \alpha)\right\},\right. \\
& \left.\quad \max \left\{{ }^{\mathrm{ABC}} D_{a+}^{\gamma} \underline{x}(t, \alpha),{ }^{\mathrm{ABC}} D_{a+}^{\gamma} \bar{x}(t, \alpha)\right\}\right],
\end{aligned}
$$

where ${ }^{\mathrm{ABC}} D_{a+}^{\gamma} \underline{x}(t, \alpha)$ and ${ }^{\mathrm{ABC}} D_{a+}^{\gamma} \bar{x}(t, \alpha)$ defined in (19).

Proof. Let the real-valued functions $\underline{x}(t, \alpha)$ and $\bar{x}(t, \alpha)$ be Atangana-Baleanu-differentiable in the Caputo sense at $t \in$ $[a, b]$, which means that $\underline{x}(t, \alpha)$ and $\bar{x}(t, \alpha)$ are differentiable at $t \in[a, b]$. From case (a) of Theorem 7 , we have that $\tilde{x}$ is gH-differentiable; then, by Definition 19 , we have that $\tilde{x}$ is $[\mathrm{gH}]_{\gamma}^{\mathrm{ABC}}$-differentiable. Using case (a) of Theorem 7, for $a<$ $\tau<t, 1-\gamma>0$, we have

$$
\begin{aligned}
& {\left[{ }_{\mathrm{gH}}^{\mathrm{ABC}} D_{a+}^{\gamma} \tilde{x}(t)\right]^{\alpha}=\frac{B(\gamma)}{1-\gamma} \int_{a}^{t} E_{\gamma}\left(-\frac{\gamma(t-\tau)^{\gamma}}{1-\gamma}\right)} \\
& \cdot\left[\min \left\{\underline{x}^{\prime}(\tau, \alpha), \bar{x}^{\prime}(\tau, \alpha)\right\},\right. \\
& \left.\max \left\{\underline{x}^{\prime}(\tau, \alpha), \bar{x}^{\prime}(\tau, \alpha)\right\}\right] d \tau=\left[\frac{B(\gamma)}{1-\gamma}\right. \\
& \cdot \min \left\{\int_{a}^{t} E_{\gamma}\left(-\frac{\gamma(t-\tau)^{\gamma}}{1-\gamma}\right) \underline{x}^{\prime}(\tau, \alpha) d \tau,\right. \\
& \left.\int_{a}^{t} E_{\gamma}\left(-\frac{\gamma(t-\tau)^{\gamma}}{1-\gamma}\right) \bar{x}^{\prime}(\tau, \alpha) d \tau\right\}, \frac{B(\gamma)}{1-\gamma} \\
& \cdot \max \left\{\int_{a}^{t} E_{\gamma}\left(-\frac{\gamma(t-\tau)^{\gamma}}{1-\gamma}\right) \underline{x}^{\prime}(\tau, \alpha) d \tau,\right. \\
& \left.\left.\int_{a}^{t} E_{\gamma}\left(-\frac{\gamma(t-\tau)^{\gamma}}{1-\gamma}\right) \bar{x}^{\prime}(\tau, \alpha) d \tau\right\}\right] \\
& =\left[\min \left\{{ }^{\mathrm{ABC}} D_{a+}^{\gamma} \underline{x}(t, \alpha),{ }^{\mathrm{ABC}} D_{a+}^{\gamma} \bar{x}(t, \alpha)\right\},\right. \\
& \left.\max \left\{{ }^{\mathrm{ABC}} D_{a+}^{\gamma} \underline{x}(t, \alpha),{ }^{\mathrm{ABC}} D_{a+}^{\gamma} \bar{x}(t, \alpha)\right\}\right] .
\end{aligned}
$$

The proof is complete.

Remark 21. According to Remark 9, a gH-differentiable fuzzy function $\tilde{x}_{\alpha}(t)=[\underline{x}(t, \alpha), \bar{x}(t, \alpha)]: K \rightarrow \mathscr{R}_{f}$, the endpoint functions $\underline{x}(t, \alpha)$ and $\bar{x}(t, \alpha)$ are not necessarily differentiable. So, the endpoint functions $\underline{x}(t, \alpha)$ and $\bar{x}(t, \alpha)$ are also not necessarily Atangana-Baleanu-differentiable in the Caputo sense. For example [25], we consider the fuzzy mapping $\tilde{x}: K \rightarrow$ $\mathscr{R}_{f}$ defined by $x(t, \alpha)=C \cdot(t-1)$, where $C$ is a fuzzy interval defined via its $\alpha$-levels by $[C]^{\alpha}=[1+\alpha, 2(3-\alpha)]$. It is well known that, in this case, $\widetilde{x}$ is a generalization of a linear function. Then,

$$
\begin{aligned}
& x(t, \alpha) \\
& \quad= \begin{cases}{[(1+\alpha)(t-1), 2(3-\alpha)(t-1)]} & \text { if } t \geq 1, \\
{[2(3-\alpha)(t-1),(1+\alpha)(t-1)]} & \text { if } 0<t<1 .\end{cases}
\end{aligned}
$$

We can see that the endpoint functions $\underline{x}(t, \alpha)$ and $\bar{x}(t, \alpha)$ are not necessarily differentiable at $t=1$. So, $\underline{x}(t, \alpha)$ and $\bar{x}(t, \alpha)$ are not necessarily Atangana-Baleanu-differentiable in the Caputo sense at $t=1$. However, $x(t, \alpha)$ is gH-differentiable and $x^{\prime}(t, \alpha)=C$ for all $t>0$.

Theorem 22. Let $D_{g H} \widetilde{x}(t) \in C\left([a, b], \mathscr{R}_{f}\right) \cap L\left([a, b], \mathscr{R}_{f}\right)$ be such that $[x(t)]^{\alpha}=[\underline{x}(t, \alpha), \bar{x}(t, \alpha)]$ for $t \in[a, b]$ and $0 \leq \alpha \leq 1$. If $(\underline{x})_{-}^{\prime}(t, \alpha),(\underline{x})_{+}^{\prime}(t, \alpha),(\bar{x})_{-}^{\prime}(t, \alpha)$, and $(\bar{x})_{+}^{\prime}(t, \alpha)$ exist and these satisfied $(\underline{x})_{-}^{\prime}(t, \alpha)=(\bar{x})_{+}^{\prime}(t, \alpha)$ and $(\underline{x})_{+}^{\prime}(t, \alpha)=$ 
$(\bar{x})_{-}^{\prime}(t, \alpha)$ at $t \in[a, b]$, then the fuzzy function $\tilde{x}(t)$ is $[g H]_{\gamma}^{\mathrm{ABC}}$ differentiable at $t \in[a, b]$ and

$$
\begin{aligned}
& {\left[{ }_{g H}^{\mathrm{ABC}} D_{a+}^{\gamma} \tilde{x}(t)\right]^{\alpha}} \\
& =\left[\min \left\{{ }_{+}^{\mathrm{ABC}} D_{a+}^{\gamma} \underline{x}(t, \alpha),{ }_{+}^{\mathrm{ABC}} D_{a+}^{\gamma} \bar{x}(t, \alpha)\right\},\right. \\
& \left.\max \left\{{ }_{+}^{\mathrm{ABC}} D_{a+}^{\gamma} \underline{x}(t, \alpha),{ }_{+}^{\mathrm{ABC}} D_{a+}^{\gamma} \bar{x}(t, \alpha)\right\}\right],
\end{aligned}
$$

where

$$
\begin{aligned}
& { }_{+}^{\mathrm{ABC}} D_{a+\underline{x}}^{\gamma}(t, \alpha) \\
& =\frac{B(\gamma)}{1-\gamma} \int_{a}^{t} E_{\gamma}\left(-\frac{\gamma(t-\tau)^{\gamma}}{1-\gamma}\right)(\underline{x})_{+}^{\prime}(\tau, \alpha) d \tau, \\
& { }_{+}^{\mathrm{ABC}} D_{a+}^{\gamma} \bar{x}(t, \alpha) \\
& =\frac{B(\gamma)}{1-\gamma} \int_{a}^{t} E_{\gamma}\left(-\frac{\gamma(t-\tau)^{\gamma}}{1-\gamma}\right)(\bar{x})_{+}^{\prime}(\tau, \alpha) d \tau .
\end{aligned}
$$

Proof. Let $(\underline{x})_{-}^{\prime}(t, \alpha),(\underline{x})_{+}^{\prime}(t, \alpha),(\bar{x})_{-}^{\prime}(t, \alpha)$, and $(\bar{x})_{+}^{\prime}(t, \alpha)$ exist, and these satisfy $(\underline{x})_{-}^{\prime}(t, \alpha)=(\bar{x})_{+}^{\prime}(t, \alpha)$ and $(\underline{x})_{+}^{\prime}(t, \alpha)=$ $(\bar{x})_{-}^{\prime}(t, \alpha)$ at $t \in[a, b]$. From case (b) of Theorem 7 , we have that $\tilde{x}$ is gH-differentiable; then, by Definition 19 , we have that $\tilde{x}$ is $[\mathrm{gH}]_{\gamma}^{\mathrm{ABC}}$-differentiable. Using case (b) of Theorem 7 , for $a<\tau<t, 1-\gamma>0$, we have

$$
\begin{aligned}
& {\left[D_{\mathrm{gH}} \tilde{x}(t)\right]^{\alpha}=\left[\min \left\{(\underline{x})_{-}^{\prime}(t, \alpha),(\bar{x})_{-}^{\prime}(t, \alpha)\right\},\right.} \\
& \left.\max \left\{(\underline{x})_{-}^{\prime}(t, \alpha),(\bar{x})_{-}^{\prime}(t, \alpha)\right\}\right]=\left[\operatorname { m i n } \left\{(\underline{x})_{+}^{\prime}(t, \alpha),\right.\right. \\
& \left.\left.(\bar{x})_{+}^{\prime}(t, \alpha)\right\}, \max \left\{(\underline{x})_{+}^{\prime}(t, \alpha),(\bar{x})_{+}^{\prime}(t, \alpha)\right\}\right], \\
& {\left[{ }_{\mathrm{gH}}^{\mathrm{ABC}} D_{a+}^{\gamma} \tilde{x}(t)\right]^{\alpha}=\frac{B(\gamma)}{1-\gamma} \int_{a}^{t} E_{\gamma}\left(-\frac{\gamma(t-\tau)^{\gamma}}{1-\gamma}\right)} \\
& \cdot\left[D_{\mathrm{gH}} x(\tau)\right]^{\alpha} d \tau=\frac{B(\gamma)}{1-\gamma} \\
& \cdot \int_{a}^{t} E_{\gamma}\left(-\frac{\gamma(t-\tau)^{\gamma}}{1-\gamma}\right) \\
& \cdot\left[\min \left\{(\underline{x})_{+}^{\prime}(\tau, \alpha),(\bar{x})_{+}^{\prime}(\tau, \alpha)\right\},\right. \\
& \left.\max \left\{(\underline{x})_{+}^{\prime}(\tau, \alpha),(\bar{x})_{+}^{\prime}(\tau, \alpha)\right\}\right] d \tau=\left[\frac{B(\gamma)}{1-\gamma}\right. \\
& \cdot \min \left\{\int_{a}^{t} E_{\gamma}\left(-\frac{\gamma(t-\tau)^{\gamma}}{1-\gamma}\right)(\underline{x})_{+}^{\prime}(\tau, \alpha) d \tau,\right. \\
& \left.\int_{a}^{t} E_{\gamma}\left(-\frac{\gamma(t-\tau)^{\gamma}}{1-\gamma}\right)(\bar{x})_{+}^{\prime}(\tau, \alpha) d \tau\right\}, \frac{B(\gamma)}{1-\gamma}
\end{aligned}
$$

$$
\begin{aligned}
& \cdot \max \left\{\int_{a}^{t} E_{\gamma}\left(-\frac{\gamma(t-\tau)^{\gamma}}{1-\gamma}\right)(\underline{x})_{+}^{\prime}(\tau, \alpha) d \tau,\right. \\
& \left.\left.\int_{a}^{t} E_{\gamma}\left(-\frac{\gamma(t-\tau)^{\gamma}}{1-\gamma}\right)(\bar{x})_{+}^{\prime}(\tau, \alpha) d \tau\right\}\right] \\
& =\left[\min \left\{{ }_{+}^{\mathrm{ABC}} D_{a+}^{\gamma} \underline{x}(t, \alpha),{ }_{+}^{\mathrm{ABC}} D_{a+}^{\gamma} \bar{x}(t, \alpha)\right\},\right. \\
& \left.\max \left\{{ }_{+}^{\mathrm{ABC}} D_{a+}^{\gamma} \underline{x}(t, \alpha),{ }_{+}^{\mathrm{ABC}} D_{a+}^{\gamma} \bar{x}(t, \alpha)\right\}\right] .
\end{aligned}
$$

The proof is complete.

Definition 23. Let $\tilde{x}:[a, b] \rightarrow \mathscr{R}_{f}$ be $[\mathrm{gH}]_{\gamma}^{\mathrm{ABC}}$-differentiable at $t \in(a, b)$. If $\underline{x}(t, \alpha)$ and $\bar{x}(t, \alpha)$ are differentiable at $t \in(a, b)$, one says that $\tilde{x}$ is $[(1)-\mathrm{gH}]_{\gamma}^{\mathrm{ABC}}$-differentiable at $t$ if

$$
\text { (1) } \begin{aligned}
& {\left[{ }_{\mathrm{gH}}^{\mathrm{ABC}} D_{a+}^{\gamma} \tilde{x}(t)\right]^{\alpha} } \\
= & {\left[{ }^{\mathrm{ABC}} D_{a+}^{\gamma} \underline{x}(t, \alpha),{ }^{\mathrm{ABC}} D_{a+}^{\gamma} \bar{x}(t, \alpha)\right], \quad 0 \leq \alpha \leq 1, }
\end{aligned}
$$

and that $\tilde{x}$ is $[(2)-\mathrm{gH}]_{\gamma}^{\mathrm{ABC}}$-differentiable at $t \in(a, b)$ if

$$
\text { (2) } \begin{aligned}
& {\left[{ }_{\mathrm{gH}}^{\mathrm{ABC}} D_{a+}^{\gamma} \tilde{x}(t)\right]^{\alpha} } \\
= & {\left[{ }^{\mathrm{ABC}} D_{a+}^{\gamma} \bar{x}(t, \alpha),{ }^{\mathrm{ABC}} D_{a+\underline{x}}^{\gamma}(t, \alpha)\right], \quad 0 \leq \alpha \leq 1, }
\end{aligned}
$$

where

$$
\begin{aligned}
{ }^{\mathrm{ABC}} D_{a+}^{\gamma} \underline{x}(t, \alpha) \\
=\frac{B(\gamma)}{1-\gamma} \int_{a}^{t} E_{\gamma}\left(-\frac{\gamma(t-\tau)^{\gamma}}{1-\gamma}\right) \frac{d}{d \tau} \underline{x}(\tau, \alpha) d \tau, \\
{ }^{\mathrm{ABC}} D_{a+}^{\gamma} \bar{x}(t, \alpha) \\
=\frac{B(\gamma)}{1-\gamma} \int_{a}^{t} E_{\gamma}\left(-\frac{\gamma(t-\tau)^{\gamma}}{1-\gamma}\right) \frac{d}{d \tau} \bar{x}(\tau, \alpha) d \tau .
\end{aligned}
$$

If $\underline{x}(t, \alpha)$ and $\bar{x}(t, \alpha)$ are not differentiable at $t \in(a, b)$, but $(\underline{x})_{-}^{\prime}(t, \alpha),(\underline{x})_{+}^{\prime}(t, \alpha),(\bar{x})_{-}^{\prime}(t, \alpha)$, and $(\bar{x})_{+}^{\prime}(t, \alpha)$ exist and these satisfy $(\underline{x})_{-}^{\prime}(t, \alpha)=(\bar{x})_{+}^{\prime}(t, \alpha)$ and $(\underline{x})_{+}^{\prime}(t, \alpha)=(\bar{x})_{-}^{\prime}(t, \alpha)$ at $t$, we say that $\tilde{x}$ is $[(3)-\mathrm{gH}]_{\gamma}^{\mathrm{ABC}}$-differentiable at $t$ if

$$
\text { (3) } \begin{aligned}
& {\left[{ }_{\mathrm{gH}}^{\mathrm{ABC}} D_{a+}^{\gamma} \tilde{x}(t)\right]^{\alpha} } \\
= & {\left[{ }_{+}^{\mathrm{ABC}} D_{a+}^{\gamma} \underline{x}(t, \alpha),{ }_{+}^{\mathrm{ABC}} D_{a+}^{\gamma} \bar{x}(t, \alpha)\right], \quad 0 \leq \alpha \leq 1, }
\end{aligned}
$$

and that $\tilde{x}$ is $[(4) \text {-gH }]_{\gamma}^{\mathrm{ABC}}$-differentiable at $t \in(a, b)$ if

$$
\text { (4) } \begin{aligned}
& {\left[{ }_{\mathrm{gH}}^{\mathrm{ABC}} D_{a+}^{\gamma} \tilde{x}(t)\right]^{\alpha} } \\
= & {\left[{ }_{+}^{\mathrm{ABC}} D_{a+}^{\gamma} \bar{x}(t, \alpha),{ }_{+}^{\mathrm{ABC}} D_{a+}^{\gamma} \underline{x}(t, \alpha)\right], \quad 0 \leq \alpha \leq 1, }
\end{aligned}
$$

where ${ }_{+}^{\mathrm{ABC}} D_{a+}^{\gamma} \bar{x}(t, \alpha)$ and ${ }_{+}^{\mathrm{ABC}} D_{a+}^{\gamma} \underline{x}(t, \alpha)$ as in Theorem 22. 
Definition 24 (see [27]). Let $\tilde{x}:[a, b] \rightarrow \mathscr{R}_{f}$ be a fuzzy function. A point $t \in(a, b)$ is said to be a switching point for the $[\mathrm{gH}]_{\gamma}^{\mathrm{ABC}}$-differentiability of $x$, if in any neighborhood $V$ of $t$ there exist points $t_{1}<t<t_{2}$ such that

type (I) at $t_{1}$ (37) holds while (38) does not hold and at $t_{2}$ (38) holds and (37) does not hold, or

type (II) at $t_{1}$ (38) holds while (37) does not hold and at $t_{2}$ (37) holds and (38) does not hold.

Theorem 25. Let $\tilde{x}:[a, b] \rightarrow \mathscr{R}_{f}$ be a fuzzy function on $[a, b]$ :

(i) If $\tilde{x}$ is [(1)-gH]-differentiable at $t \in[a, b]$, then $\left[{ }_{g H}^{\mathrm{ABC}} D_{a+}^{\gamma} \tilde{x}(t)\right]^{\alpha}=\left[{ }^{\mathrm{ABC}} D_{a+}^{\gamma} \underline{x}(t, \alpha),{ }^{\mathrm{ABC}} D_{a+}^{\gamma} \bar{x}(t, \alpha)\right]$.

(ii) If $\tilde{x}$ is [(2)-gH]-differentiable at $t \in[a, b]$, then $\left[{ }_{g H}^{\mathrm{ABC}} D_{a+}^{\gamma} \tilde{x}(t)\right]^{\alpha}=\left[{ }^{\mathrm{ABC}} D_{a+}^{\gamma} \bar{x}(t, \alpha),{ }^{\mathrm{ABC}} D_{a+}^{\gamma} \underline{x}(t, \alpha)\right]$.

Proof. The proof is similar to [27].

\section{The Necessary Optimality Conditions for Fuzzy Fractional Variational Problems}

In this section, we consider the following fuzzy fractional variational problem (FFVP) with the Atangana-Baleanu fractional derivative in the Caputo sense. Let the fuzzy function $\tilde{x}(t)=[\underline{x}(t, \alpha), \bar{x}(t, \alpha)] \in C^{1}\left([0, b], \mathscr{R}_{f}\right) \cap L\left([0, b], \mathscr{R}_{f}\right):$

$$
\min \widetilde{J}(\tilde{x})=\int_{0}^{b} \widetilde{L}\left(t, \widetilde{x}(t),{ }_{\mathrm{gH}}^{\mathrm{ABC}} D_{i 0+}^{\gamma} \widetilde{x}(t)\right) d t
$$

with $\tilde{x}(0)=\tilde{x}_{0}$ and $\tilde{x}(b)=\tilde{x}_{b}$ and $i=1,2$, where $\tilde{x}_{0}, \tilde{x}_{b} \in \mathscr{R}_{f}$. The assumptions are as follows:

(1) $\widetilde{L}:[0, b] \times \mathscr{R}_{f}^{2} \rightarrow \mathscr{R}_{f}$ is continuously differentiable with respect to all its arguments, which means $\underline{L}$ and $\bar{L} \epsilon$ $C^{1}\left([0, b], \mathscr{R}_{f}\right)$, and the first variation of the functional $\widetilde{L}$ must be vanished.

(2) Given any $\widetilde{x}$, the map $t \mapsto{ }_{\mathrm{gH}}^{\mathrm{ABC}} D_{b_{-}}^{\beta}\left(\partial_{3} \widetilde{L}\right)$ is continuous.

Hereafter, we denote by $\partial_{i} L$ the partial derivative of the function $L$ with respect to its $i$ th argument. The fuzzy function $\tilde{x}(t)$ is $[(i)-\mathrm{gH}]_{\gamma}^{\mathrm{ABC}}$-differentiable with respect to the independent variable $t$ for $i \in\{1,2\}$, which is denoted as ${ }_{\mathrm{gH}}^{\mathrm{ABC}} D_{i 0+}^{\gamma} \tilde{x}(t)$. We consider $\gamma \in(0,1) ;{ }_{\mathrm{gH}}^{\mathrm{ABC}} D_{i 0+}^{\gamma} \tilde{x}(t)$ denotes the Atangana-Baleanu fuzzy fractional derivative as follows:

$$
\begin{aligned}
& { }_{\mathrm{gH}}^{\mathrm{ABC}} D_{i 0+}^{\gamma} \tilde{x}(t) \\
& =\frac{B(\gamma)}{1-\gamma} \int_{0}^{t} E_{\gamma}\left(-\frac{\gamma(t-\tau)^{\gamma}}{1-\gamma}\right) D_{i, \mathrm{gH}} \tilde{x}(\tau) d \tau,
\end{aligned}
$$

for $i=1,2$.

Theorem 26. Assume that $\tilde{x} \in C^{1}\left([0, b], \mathscr{R}_{f}\right)$. Then, its fuzzy $g H$-Atangana-Baleanu fractional derivative in the Caputo sense is continuous on the closed interval $[0, b]$.
Proof. From Definition 19, the fuzzy gH-Atangana-Baleanu fractional derivative in the Caputo sense of $\tilde{x}$ is defined as follows:

$$
\begin{aligned}
& { }_{\mathrm{gH}}^{\mathrm{ABC}} D_{a+}^{\gamma} \tilde{x}(t) \\
& \quad=\frac{B(\gamma)}{1-\gamma} \int_{a}^{t} E_{\gamma}\left(-\frac{\gamma(t-\tau)^{\gamma}}{1-\gamma}\right)\left(D_{\mathrm{gH}} \tilde{x}\right)(\tau) d \tau,
\end{aligned}
$$

where $0<\gamma \leq 1, t>a$, and $a=0$.

Since $\tilde{x} \in C^{1}\left([0, b], \mathscr{R}_{f}\right), D_{\mathrm{gH}} \tilde{x}$ is continuous on the closed interval $[0, b]$. It can be shown that ${ }_{\mathrm{gH}}^{\mathrm{ABC}} D_{a+}^{\gamma} \tilde{x}(t)$ is also continuous on the closed interval $[0, b]$.

Similar to [22], we define the concepts of admissible curve and fuzzy weak neighborhood under the assumption of fuzzy gH-Atangana-Baleanu fractional differentiability.

Definition 27. One says that the fuzzy curve $\tilde{x}(t)=$ $[\underline{x}(t, \alpha), \bar{x}(t, \alpha)]$ is admissible if it satisfies the end conditions and the two functions $\underline{x}(t, \alpha)$ and $\bar{x}(t, \alpha)$ have a continuous Atangana-Baleanu fractional derivative in the Caputo sense of order $\gamma,(0<\gamma, \alpha<1)$ in $[0, b]$. One denotes the set of all admissible curves by $\widetilde{X}_{0 d}$.

Definition 28. A fuzzy weak neighborhood $\widetilde{N}_{\varepsilon}(\tilde{x})$ of a fuzzy curve $\widetilde{\hat{x}}=\widetilde{\widetilde{x}}(t)$ is the set of all admissible curves $\tilde{x} \in \widetilde{X}_{0 d}$ satisfying, for all $t \in[0, b]$,

$$
\begin{aligned}
d(\tilde{x}(t), \widetilde{\widetilde{x}}(t)) \leq \varepsilon, & \forall t \in[0, b], \\
d\left({ }_{\mathrm{gH}}^{\mathrm{ABC}} D_{i 0+}^{\gamma} \widetilde{x}(t),{ }_{\mathrm{gH}} D_{i 0+}^{\gamma} \widetilde{\widetilde{x}}(t)\right) \leq \varepsilon, & \forall t \in[0, b],
\end{aligned}
$$

where $\varepsilon$ is a small positive real number and $i=1,2$.

For the problem of (FFVP), it is to find the admissible curves $\tilde{x} \in \widetilde{X}_{0 d}$ in a fuzzy weak neighborhood, if any exists, such that $\widetilde{J}$ is minimized. Let $\tilde{x}^{*}=\tilde{x}^{*}(t)$ be a minimized fuzzy curve of (FFVP), which means

$$
\widetilde{J}(\widetilde{x}) \geq \widetilde{J}\left(\tilde{x}^{*}\right)
$$

for all admissible curves $\tilde{x} \in \widetilde{X}_{0 d}$ in the fuzzy weak neighborhood $\widetilde{N}_{\varepsilon}\left(\tilde{x}^{*}\right)$. According to Definition 4 ,

$$
\begin{aligned}
& \underline{J}^{\alpha}(\tilde{x}) \geq \underline{J}^{\alpha}\left(\tilde{x}^{*}\right), \\
& \bar{J}^{\alpha}(\tilde{x}) \geq \bar{J}^{\alpha}\left(\tilde{x}^{*}\right)
\end{aligned}
$$

for all $\alpha \in[0,1]$ and all admissible curves $\tilde{x} \in \widetilde{X}_{0 d}$ in $\widetilde{N}_{\varepsilon}\left(\widetilde{x}^{*}\right)$.

Proposition 29. Let $\tilde{x}(t)$ and $\tilde{h}(t)$ be all admissible curves in $\widetilde{X}_{0 d}$. Then, for $k \in \mathbf{R}_{+}$and $\gamma \in[0,1]$, 


$$
\begin{aligned}
& { }_{g H}^{\mathrm{ABC}} D_{0+}^{\gamma}(\tilde{x}(t)+k \tilde{h}(t))=\left[\min \left\{{ }^{\mathrm{ABC}} D_{0+}^{\gamma}(\underline{x}(t, \alpha)+k \underline{h}(t, \alpha)),{ }^{\mathrm{ABC}} D_{0+}^{\gamma}(\bar{x}(t, \alpha)+k \bar{h}(t, \alpha))\right\},\right. \\
& \left.\max \left\{{ }^{\mathrm{ABC}} D_{0+}^{\gamma}(\underline{x}(t, \alpha)+k \underline{h}(t, \alpha)),{ }^{\mathrm{ABC}} D_{0+}^{\gamma}(\bar{x}(t, \alpha)+k \bar{h}(t, \alpha))\right\}\right] \\
& =\left[\min \left\{{ }^{\mathrm{ABC}} D_{0+}^{\gamma} \underline{x}(t, \alpha)+k^{\mathrm{ABC}} D_{0+}^{\gamma} \underline{h}(t, \alpha),{ }^{\mathrm{ABC}} D_{0+}^{\gamma} \bar{x}(t, \alpha)+k^{\mathrm{ABC}} D_{0+}^{\gamma} \bar{h}(t, \alpha)\right\},\right. \\
& \left.\max \left\{{ }^{\mathrm{ABC}} D_{0+}^{\gamma} \underline{x}(t, \alpha)+k^{\mathrm{ABC}} D_{0+}^{\gamma} \underline{h}(t, \alpha),{ }^{\mathrm{ABC}} D_{0+}^{\gamma} \bar{x}(t, \alpha)+k^{\mathrm{ABC}} D_{0+}^{\gamma} \bar{h}(t, \alpha)\right\}\right] .
\end{aligned}
$$

Proof. According to the assumptions, $\tilde{x}(t)$ and $\tilde{h}(t)$ are all admissible curves in $\widetilde{X}_{0 d}$ and the fuzzy functions $\widetilde{x}(t)$ and $\tilde{h}(t)$ are $[(i)-\mathrm{gH}]_{\gamma}^{\mathrm{ABC}}$-differentiable with respect to the independent variable $t$ for $i \in\{1,2\}$.

From Definitions 23 and 27, $\underline{x}(t, \alpha), \underline{h}(t, \alpha), \bar{x}(t, \alpha)$, and $\bar{h}(t, \alpha)$ are differentiable at $t \in(0, \bar{b})$. So, by Definition 19 , we have

$$
\begin{aligned}
& { }_{\mathrm{gH}}^{\mathrm{ABC}} D_{0+}^{\gamma}(\tilde{x}(t)+k \widetilde{h}(t)) \\
& =\frac{B(\gamma)}{1-\gamma} \int_{0}^{t} E_{\gamma}\left(-\frac{\gamma(t-\tau)^{\gamma}}{1-\gamma}\right)\left(D_{\mathrm{gH}}(\tilde{x}+k \tilde{h})\right)(\tau) d \tau .
\end{aligned}
$$

Since $k \in \mathbf{R}_{+}$, we get

$$
\begin{aligned}
& {[\tilde{x}(t)+k \tilde{h}(t)]^{\alpha}} \\
& \quad=[\underline{x}(t, \alpha)+k \underline{h}(t, \alpha), \bar{x}(t, \alpha)+k \bar{h}(t, \alpha)] .
\end{aligned}
$$

Using Theorem $7(\mathrm{a})$, we have

$$
\begin{aligned}
& {\left[D_{\mathrm{gH}}(\tilde{x}+k \tilde{h})(\tau)\right]^{\alpha}=\left[\operatorname { m i n } \left\{\underline{x}^{\prime}(\tau, \alpha)\right.\right.} \\
& \left.\quad+k \underline{h}^{\prime}(\tau, \alpha), \bar{x}^{\prime}(\tau, \alpha)+k \bar{h}^{\prime}(t, \alpha)\right\}, \max \left\{\underline{x}^{\prime}(\tau, \alpha)\right. \\
& \left.\left.\quad+k \underline{h}^{\prime}(\tau, \alpha), \bar{x}^{\prime}(\tau, \alpha)+k \bar{h}^{\prime}(t, \alpha)\right\}\right] .
\end{aligned}
$$

By the above equations, we obtain

$$
\begin{aligned}
& { }_{\mathrm{gH}}^{\mathrm{ABC}} D_{0+}^{\gamma}(\tilde{x}(t)+k \tilde{h}(t)) \\
& =\left[\operatorname { m i n } \left\{{ }^{\mathrm{ABC}} D_{0+}^{\gamma}(\underline{x}(t, \alpha)+k \underline{h}(t, \alpha)),\right.\right. \\
& \left.{ }^{\mathrm{ABC}} D_{0+}^{\gamma}(\bar{x}(t, \alpha)+k \bar{h}(t, \alpha))\right\}, \\
& \max \left\{{ }^{\mathrm{ABC}} D_{0+}^{\gamma}(\underline{x}(t, \alpha)+k \underline{h}(t, \alpha)),\right. \\
& \left.\left.{ }^{\mathrm{ABC}} D_{0+}^{\gamma}(\bar{x}(t, \alpha)+k \bar{h}(t, \alpha))\right\}\right] \\
& =\left[\operatorname { m i n } \left\{{ }^{\mathrm{ABC}} D_{0+}^{\gamma} \underline{x}(t, \alpha)+k^{\mathrm{ABC}} D_{0+}^{\gamma} \underline{h}(t, \alpha),\right.\right. \\
& \left.{ }^{\mathrm{ABC}} D_{0+}^{\gamma} \bar{x}(t, \alpha)+k^{\mathrm{ABC}} D_{0+}^{\gamma} \bar{h}(t, \alpha)\right\}, \\
& \max \left\{{ }^{\mathrm{ABC}} D_{0+}^{\gamma} \underline{x}(t, \alpha)+k^{\mathrm{ABC}} D_{0+}^{\gamma} \underline{h}(t, \alpha),\right. \\
& \left.\left.{ }_{\mathrm{ABC}} D_{0+}^{\gamma} \bar{x}(t, \alpha)+k^{\mathrm{ABC}} D_{0+}^{\gamma} \bar{h}(t, \alpha)\right\}\right] .
\end{aligned}
$$

To develop the necessary conditions for the problem of (FFVP), we assume that $\tilde{x}^{*}(t)$ is the desired function; let $\epsilon \in \mathbf{R}_{+}$and define a family of curves $\tilde{x}(t)=\tilde{x}^{*}(t)+\epsilon \widetilde{h}(t)$, where $\widetilde{h}(t)=[\underline{h}(t, \alpha), \bar{h}(t, \alpha)]$ is an arbitrary admissible variation and the two real-valued functions $\underline{h}(t, \alpha)$ and $\bar{h}(t, \alpha)$ are differentiable for all $\alpha \in[0,1]$. We present the necessary condition for the problem of (FFVP) as follows.

Theorem 30. Let $\tilde{x}^{*}$ be a solution of (FFVP). Then, $\tilde{x}^{*}$ is a solution of the fractional Euler-Lagrange equations

$$
\begin{aligned}
& \partial_{2} \underline{L}(Z)+{ }^{\mathrm{ABR}} D_{b_{-}}^{\gamma} \partial_{4} \underline{L}(Z)=0, \\
& \partial_{3} \underline{L}(Z)+{ }^{\mathrm{ABR}} D_{b_{-}}^{\gamma} \partial_{5} \underline{L}(Z)=0, \\
& \partial_{2} \bar{L}(Z)+{ }^{\mathrm{ABR}} D_{b_{-}}^{\gamma} \partial_{4} \bar{L}(Z)=0, \\
& \partial_{3} \bar{L}(Z)+{ }^{\mathrm{ABR}} D_{b_{-}}^{\gamma} \partial_{5} \bar{L}(Z)=0,
\end{aligned}
$$

for all $t \in[0, b]$, where

$$
\begin{gathered}
Z=\left(t, \underline{x}^{*}(t, \alpha), \bar{x}^{*}(t, \alpha),{ }^{\mathrm{ABC}} D_{i 0+}^{\gamma} \underline{x}^{*}(t, \alpha),\right. \\
\left.{ }^{\mathrm{ABC}} D_{i 0+}^{\gamma} \bar{x}^{*}(t, \alpha)\right), \quad i \in\{1,2\} .
\end{gathered}
$$

Proof. Consider $\widetilde{x}^{*}+\epsilon \widetilde{h}$ as an admissible variation for $\epsilon \in \mathbf{R}_{+}$, and $\widetilde{h}(t)=[\underline{h}(t, \alpha), \bar{h}(t, \alpha)]:[0, b] \rightarrow \mathscr{R}_{f}$ is an arbitrary admissible variation. The two real-valued functions $\underline{h}(t, \alpha)$ and $\bar{h}(t, \alpha)$ are differentiable for all $\alpha \in[0,1]$. Let $\widetilde{j}(\epsilon)=$ $\widetilde{J}\left(\widetilde{x}^{*}+\epsilon \widetilde{h}\right):$

$$
\begin{aligned}
& \widetilde{j}(\epsilon)=\int_{0}^{b} \widetilde{L}\left(t, \tilde{x}^{*}(t)\right. \\
&\left.+\epsilon \widetilde{h}(t),{ }_{\mathrm{gH}}^{\mathrm{ABC}} D_{i 0+}^{\gamma}\left(\widetilde{x}^{*}(t)+\epsilon \widetilde{h}(t)\right)\right) d t
\end{aligned}
$$

for $i=1$, 2. From Proposition 29, we have 


$$
\begin{aligned}
& { }_{\mathrm{gH}}^{\mathrm{ABC}} D_{0+}^{\gamma}\left(\tilde{x}^{*}(t)+\epsilon \tilde{h}(t)\right) \\
& =\left[\operatorname { m i n } \left\{{ }^{\mathrm{ABC}} D_{0+}^{\gamma}\left(\underline{x}^{*}(t, \alpha)+\epsilon \underline{h}(t, \alpha)\right),\right.\right. \\
& \left.{ }^{\mathrm{ABC}} D_{0+}^{\gamma}\left(\bar{x}^{*}(t, \alpha)+\epsilon \bar{h}(t, \alpha)\right)\right\}, \\
& \max \left\{{ }^{\mathrm{ABC}} D_{0+}^{\gamma}\left(\underline{x}^{*}(t, \alpha)+\epsilon \underline{h}(t, \alpha)\right),\right. \\
& \left.\left.{ }^{\mathrm{ABC}} D_{0+}^{\gamma}\left(\bar{x}^{*}(t, \alpha)+\epsilon \bar{h}(t, \alpha)\right)\right\}\right] \\
& =\left[\operatorname { m i n } \left\{{ }^{\mathrm{ABC}} D_{0+}^{\gamma} \underline{x}^{*}(t, \alpha)+\epsilon{ }^{\mathrm{ABC}} D_{0+}^{\gamma} \underline{h}(t, \alpha),\right.\right. \\
& \left.{ }_{\mathrm{ABC}} D_{0+}^{\gamma} \bar{x}^{*}(t, \alpha)+\epsilon^{\mathrm{ABC}} D_{0+}^{\gamma} \bar{h}(t, \alpha)\right\}, \\
& \max ^{\mathrm{ABC}} D_{0+}^{\gamma} \underline{x}^{*}(t, \alpha)+\epsilon^{\mathrm{ABC}} D_{0+}^{\gamma} \underline{h}(t, \alpha), \\
& \left.\left.{ }_{\mathrm{ABC}} D_{0+}^{\gamma} \bar{x}^{*}(t, \alpha)+\epsilon^{\mathrm{ABC}} D_{0+}^{\gamma} \bar{h}(t, \alpha)\right\}\right] .
\end{aligned}
$$

Then, the lower and upper bounds of $\tilde{j}$ are

$$
\begin{aligned}
& \underline{j}(\epsilon)=\int_{0}^{b} \underline{L}(\cdots)^{\alpha} d t \\
& \bar{j}(\epsilon)=\int_{0}^{b} \bar{L}(\cdots)^{\alpha} d t
\end{aligned}
$$

respectively, where

$$
\begin{aligned}
& (\cdots)^{\alpha}=\left(t, \underline{x}^{*}(t, \alpha)+\epsilon \underline{h}(t, \alpha), \bar{x}^{*}(t, \alpha)\right. \\
& +\epsilon \bar{h}(t, \alpha),{ }^{\mathrm{ABC}} D_{0+}^{\gamma} \underline{x}^{*}(t, \alpha) \\
& +\epsilon^{\mathrm{ABC}} D_{0+}^{\gamma} \underline{h}(t, \alpha),{ }^{\mathrm{ABC}} D_{0+}^{\gamma} \bar{x}^{*}(t, \alpha) \\
& \left.+\epsilon^{\mathrm{ABC}} D_{0+}^{\gamma} \bar{h}(t, \alpha)\right) .
\end{aligned}
$$

Since $\tilde{x}$ is a solution of (FFVP), then

$$
\left.\frac{d \underline{j}(\epsilon)}{d \epsilon}\right|_{\epsilon=0}=\left.\frac{d \bar{j}(\epsilon)}{d \epsilon}\right|_{\epsilon=0}=0 .
$$

Computing $\left.\underline{j}^{\prime}(\epsilon)\right|_{\epsilon=0}$, we get

$$
\begin{aligned}
& \int_{0}^{b}\left[\partial_{2} \underline{L}(Z) \underline{h}+\partial_{3} \underline{L}(Z) \bar{h}+\partial_{4} \underline{L}(Z)^{\mathrm{ABC}} D_{0+}^{\gamma} \underline{h}\right. \\
& \left.\quad+\partial_{5} \underline{L}(Z)^{\mathrm{ABC}} D_{0+}^{\gamma} \bar{h}\right] d t=0,
\end{aligned}
$$

where

$$
\begin{gathered}
Z=\left(t, \underline{x}^{*}(t, \alpha), \bar{x}^{*}(t, \alpha),{ }_{\mathrm{gH}}^{\mathrm{ABC}} D_{i 0+}^{\gamma} \underline{x}^{*}(t, \alpha),\right. \\
\left.{ }_{\mathrm{gH}}^{\mathrm{ABC}} D_{i 0+}^{\gamma} \bar{x}^{*}(t, \alpha)\right), \quad i \in\{1,2\} .
\end{gathered}
$$

Using Proposition 18, we obtain

$$
\begin{aligned}
& \int_{0}^{b} \partial_{4} \underline{L}(Z)^{\mathrm{ABC}} D_{0+}^{\gamma} \underline{h} d t \\
& =\int_{0}^{b} \underline{h}^{\mathrm{ABR}} D_{b-}^{\gamma} \partial_{4} \underline{L}(Z) d t \\
& \quad+\left.\frac{B(\gamma)}{1-\gamma}\left[\underline{h} \mathbf{E}_{\gamma, 1,-\gamma /(1-\gamma), b-}^{1} \partial_{4} \underline{L}(Z)\right]\right|_{t=0} ^{t=b}, \\
& \int_{0}^{b} \partial_{5} \underline{L}(Z)^{\mathrm{ABC}} D_{0+}^{\gamma} \bar{h} d t \\
& =\int_{0}^{b} \bar{h}^{\mathrm{ABR}} D_{b-}^{\gamma} \partial_{5} \underline{L}(Z) d t \\
& \quad+\left.\frac{B(\gamma)}{1-\gamma}\left[\bar{h} \mathbf{E}_{\gamma, 1,-\gamma /(1-\gamma), b-}^{1} \partial_{5} \underline{L}(Z)\right]\right|_{t=0} ^{t=b} .
\end{aligned}
$$

Considering the end conditions of $\underline{h}(0, \alpha)=\bar{h}(0, \alpha)=$ $\underline{h}(b, \alpha)=\bar{h}(b, \alpha)=0$ and substituting (61) into (59), then

$$
\begin{aligned}
& \int_{0}^{b}\left\{\left[\partial_{2} \underline{L}(Z)+{ }^{\mathrm{ABR}} D_{b_{-}}^{\gamma} \partial_{4} \underline{L}(Z)\right] \underline{h}\right. \\
& \left.\quad+\left[\partial_{3} \underline{L}(Z)+{ }^{\mathrm{ABR}} D_{b_{-}}^{\gamma} \partial_{5} \underline{L}(Z)\right] \bar{h}\right\} d t=0 .
\end{aligned}
$$

Since $\underline{h}(t, \alpha)$ and $\bar{h}(t, \alpha)$ are arbitrary elsewhere, we get

$$
\begin{aligned}
& \partial_{2} \underline{L}(Z)+{ }^{\mathrm{ABR}} D_{b_{-}}^{\gamma} \partial_{4} \underline{L}(Z)=0, \\
& \partial_{3} \underline{L}(Z)+{ }^{\mathrm{ABR}} D_{b_{-}}^{\gamma} \partial_{5} \underline{L}(Z)=0
\end{aligned}
$$

for all $t \in[0, b]$. Following the scheme of obtaining (59), (61), and (63) and computing $\left.\bar{j}^{\prime}(\epsilon)\right|_{\epsilon=0}=0$, we can also obtain

$$
\begin{aligned}
& \partial_{2} \bar{L}(Z)+{ }^{\mathrm{ABR}} D_{b_{-}}^{\gamma} \partial_{4} \bar{L}(Z)=0, \\
& \partial_{3} \bar{L}(Z)+{ }^{\mathrm{ABR}} D_{b_{-}}^{\gamma} \partial_{5} \bar{L}(Z)=0
\end{aligned}
$$

for all $t \in[0, b]$, where

$$
\begin{gathered}
Z=\left(t, \underline{x}^{*}(t, \alpha), \bar{x}^{*}(t, \alpha),{ }_{\mathrm{gH}}^{\mathrm{ABC}} D_{i 0+}^{\gamma} \underline{x}^{*}(t, \alpha),\right. \\
\left.{ }_{\mathrm{gH}}^{\mathrm{ABC}} D_{i 0+}^{\gamma} \bar{x}^{*}(t, \alpha)\right), \quad i \in\{1,2\} .
\end{gathered}
$$

Next, we consider a more general class of fractional variational problems, for $A \in(0, b)$ and the functional

$$
\min \widetilde{J}(\tilde{x})=\int_{A}^{b} \widetilde{L}\left(t, \tilde{x}(t),{ }_{\mathrm{gH}}^{\mathrm{ABC}} D_{i 0+}^{\gamma} \tilde{x}(t)\right) d t
$$

with $\tilde{x}(A)=\tilde{x}_{A}$ and $\tilde{x}(b)=\tilde{x}_{b}$ and $i=1,2$, where $\tilde{x}_{A}, \tilde{x}_{b} \in$ $\mathscr{R}_{f}$. The assumptions are the same as previously stated for $\widetilde{L}\left(t, \tilde{x}(t),{ }_{\mathrm{gH}}^{\mathrm{ABC}} D_{i 0+}^{\gamma} \tilde{x}(t)\right)$. 
Theorem 31. If $\tilde{x}$ is a solution of (GFFVP), then $\tilde{x}$ satisfies

$$
\begin{aligned}
& { }^{\mathrm{ABR}} D_{b-}^{\gamma} \partial_{4} \underline{L}(Z)-{ }^{\mathrm{ABR}} D_{A_{-}}^{\gamma} \partial_{4} \underline{L}(Z)=0, \\
& { }^{\mathrm{ABR}} D_{b-}^{\gamma} \partial_{5} \underline{L}(Z)-{ }^{\mathrm{ABR}} D_{A_{-}}^{\gamma} \partial_{5} \underline{L}(Z)=0, \\
& { }^{\mathrm{ABR}} D_{b_{-}}^{\gamma} \partial_{4} \bar{L}(Z)-{ }^{\mathrm{ABR}} D_{A_{-}}^{\gamma} \partial_{4} \bar{L}(Z)=0, \\
& { }^{\mathrm{ABR}} D_{b-}^{\gamma} \partial_{5} \bar{L}(Z)-{ }^{\mathrm{ABR}} D_{A_{-}}^{\gamma} \partial_{5} \bar{L}(Z)=0,
\end{aligned}
$$

on $[0, A]$,

$$
\begin{aligned}
& \partial_{2} \underline{L}(Z)+{ }^{\mathrm{ABR}} D_{b-}^{\gamma} \partial_{4} \underline{L}(Z)=0, \\
& \partial_{3} \underline{L}(Z)+{ }^{\mathrm{ABR}} D_{b-}^{\gamma} \partial_{5} \underline{L}(Z)=0, \\
& \partial_{2} \bar{L}(Z)+{ }^{\mathrm{ABR}} D_{b-}^{\gamma} \partial_{4} \bar{L}(Z)=0, \\
& \partial_{3} \bar{L}(Z)+{ }^{\mathrm{ABR}} D_{b-}^{\gamma} \partial_{5} \bar{L}(Z)=0,
\end{aligned}
$$

on $[A, b]$, and

$$
\begin{aligned}
& \mathbf{E}_{\gamma, 1,-\gamma /(1-\gamma), A-}^{1} \partial_{4} \underline{L}(Z)-\mathbf{E}_{\gamma, 1,-\gamma /(1-\gamma), b-}^{1} \partial_{4} \underline{L}(Z)=0, \\
& \mathbf{E}_{\gamma, 1,-\gamma /(1-\gamma), A-}^{1} \partial_{5} \underline{L}(Z)-\mathbf{E}_{\gamma, 1,-\gamma /(1-\gamma), b-}^{1} \partial_{5} \underline{L}(Z)=0, \\
& \mathbf{E}_{\gamma, 1,-\gamma /(1-\gamma), A-}^{1} \partial_{4} \bar{L}(Z)-\mathbf{E}_{\gamma, 1,-\gamma /(1-\gamma), b_{-}}^{1} \partial_{4} \bar{L}(Z)=0, \\
& \mathbf{E}_{\gamma, 1,-\gamma /(1-\gamma), A-}^{1} \partial_{5} \bar{L}(Z)-\mathbf{E}_{\gamma, 1,-\gamma /(1-\gamma), b-}^{1} \partial_{5} \bar{L}(Z)=0,
\end{aligned}
$$

at $t=0$.

Proof. Let $\tilde{x}^{*}$ be a solution of (GFFVP). Consider $\tilde{x}^{*}+\epsilon \widetilde{h}$ as an admissible variation for $\epsilon \in \mathbf{R}_{+}$with $|\epsilon| \ll 1$, and $\tilde{h}(t)=$ $[\underline{h}(t, \alpha), \bar{h}(t, \alpha)]:[0, b] \rightarrow \mathscr{R}_{f}$ is an arbitrary admissible variation. The two real-valued functions $\underline{h}(t, \alpha)$ and $\bar{h}(t, \alpha)$ are differentiable for all $\alpha \in[0,1]$. Let $\widetilde{j}(\epsilon)=\widetilde{J}\left(\widetilde{x}^{*}+\epsilon \widetilde{h}\right)$ :

$$
\begin{aligned}
& \tilde{j}(\epsilon)=\int_{A}^{b} \widetilde{L}\left(t, \tilde{x}^{*}(t)\right. \\
&\left.+\epsilon \tilde{h}(t),{ }_{\mathrm{gH}}^{\mathrm{ABC}} D_{i 0+}^{\gamma}\left(\tilde{x}^{*}(t)+\epsilon \widetilde{h}(t)\right)\right) d t
\end{aligned}
$$

for $i=1$, 2. From Proposition 29, we have

$$
\begin{aligned}
& { }_{\mathrm{gH}}^{\mathrm{ABC}} D_{0+}^{\gamma}\left(\tilde{x}^{*}(t)+\epsilon \widetilde{h}(t)\right)=\left[\operatorname { m i n } \left\{{ }^{\mathrm{ABC}} D_{0+}^{\gamma} \underline{x}^{*}(t, \alpha)\right.\right. \\
& +\epsilon^{\mathrm{ABC}} D_{0+}^{\gamma} \underline{h}(t, \alpha),{ }^{\mathrm{ABC}} D_{0+}^{\gamma} \bar{x}^{*}(t, \alpha) \\
& \left.+\epsilon^{\mathrm{ABC}} D_{0+}^{\gamma} \bar{h}(t, \alpha)\right\}, \max \left\{{ }^{\mathrm{ABC}} D_{0+}^{\gamma} \underline{x}^{*}(t, \alpha)\right. \\
& +\epsilon^{\mathrm{ABC}} D_{0+}^{\gamma} \underline{h}(t, \alpha),{ }^{\mathrm{ABC}} D_{0+}^{\gamma} \bar{x}^{*}(t, \alpha) \\
& \left.\left.+\epsilon^{\mathrm{ABC}} D_{0+}^{\gamma} \bar{h}(t, \alpha)\right\}\right] .
\end{aligned}
$$

The lower and upper bounds of $\tilde{j}$ are

$$
\begin{aligned}
& \underline{j}(\epsilon)=\int_{A}^{b} \underline{L}(\cdots)^{\alpha} d t, \\
& \bar{j}(\epsilon)=\int_{A}^{b} \bar{L}(\cdots)^{\alpha} d t,
\end{aligned}
$$

respectively, where

$$
\begin{aligned}
& (\cdots)^{\alpha}=\left(t, \underline{x}^{*}(t, \alpha)+\epsilon \underline{h}(t, \alpha), \bar{x}^{*}(t, \alpha)\right. \\
& +\epsilon \bar{h}(t, \alpha),{ }^{\mathrm{ABC}} D_{0+}^{\gamma} \underline{x}^{*}(t, \alpha) \\
& +\epsilon^{\mathrm{ABC}} D_{0+}^{\gamma} \underline{h}(t, \alpha),{ }^{\mathrm{ABC}} D_{0+}^{\gamma} \bar{x}^{*}(t, \alpha) \\
& \left.+\epsilon^{\mathrm{ABC}} D_{0+}^{\gamma} \bar{h}(t, \alpha)\right) .
\end{aligned}
$$

Since $\tilde{x}$ is a solution of (GFFVP), then

$$
\left.\frac{d \underline{j}(\epsilon)}{d \epsilon}\right|_{\epsilon=0}=\left.\frac{d \bar{j}(\epsilon)}{d \epsilon}\right|_{\epsilon=0}=0 .
$$

Computing $\left.\underline{j}^{\prime}(\epsilon)\right|_{\epsilon=0}$, we get

$$
\begin{aligned}
& \int_{A}^{b}\left[\partial_{2} \underline{L}(Z) \underline{h}+\partial_{3} \underline{L}(Z) \bar{h}+\partial_{4} \underline{L}(Z)^{\mathrm{ABC}} D_{0+}^{\gamma} \underline{h}\right. \\
& \left.+\partial_{5} \underline{L}(Z){ }^{\mathrm{ABC}} D_{0+}^{\gamma} \bar{h}\right] d t=\int_{A}^{b}\left[\partial_{2} \underline{L}(Z) \underline{h}\right. \\
& \left.+\partial_{3} \underline{L}(Z) \bar{h}\right] d t+\int_{0}^{b}\left[\partial_{4} \underline{L}(Z){ }^{\mathrm{ABC}} D_{0+}^{\gamma} \underline{h}\right. \\
& \left.+\partial_{5} \underline{L}(Z){ }^{\mathrm{ABC}} D_{0+}^{\gamma} \bar{h}\right] d t \\
& -\int_{0}^{A}\left[\partial_{4} \underline{L}(Z){ }^{\mathrm{ABC}} D_{0+}^{\gamma} \underline{h}+\partial_{5} \underline{L}(Z){ }^{\mathrm{ABC}} D_{0+}^{\gamma} \bar{h}\right] d t \\
& =\int_{A}^{b}\left[\partial_{2} \underline{L}(Z) \underline{h}+\partial_{3} \underline{L}(Z) \bar{h}\right] d t \\
& +\int_{0}^{b} \underline{h}^{\mathrm{ABR}} D_{b_{-}}^{\gamma} \partial_{4} \underline{L}(Z) d t \\
& +\left.\frac{B(\gamma)}{1-\gamma}\left[\underline{h} \mathbf{E}_{\gamma, 1,-\gamma /(1-\gamma), b-}^{1} \partial_{4} \underline{L}(Z)\right]\right|_{t=0} ^{t=b} \\
& +\int_{0}^{b} \bar{h}^{\mathrm{ABR}} D_{b_{-}}^{\gamma} \partial_{5} \underline{L}(Z) d t \\
& +\left.\frac{B(\gamma)}{1-\gamma}\left[\bar{h} \mathbf{E}_{\gamma, 1,-\gamma /(1-\gamma), b-}^{1} \partial_{5} \underline{L}(Z)\right]\right|_{t=0} ^{t=b} \\
& -\int_{0}^{A} \underline{h}^{\mathrm{ABR}} D_{A-}^{\gamma} \partial_{4} \underline{L}(Z) d t \\
& -\left.\frac{B(\gamma)}{1-\gamma}\left[\underline{h} \mathbf{E}_{\gamma, 1,-\gamma /(1-\gamma), A-}^{1} \partial_{4} \underline{L}(Z)\right]\right|_{t=0} ^{t=A} \\
& -\int_{0}^{A} \bar{h}^{\mathrm{ABR}} D_{A-}^{\gamma} \partial_{5} \underline{L}(Z) d t \\
& -\left.\frac{B(\gamma)}{1-\gamma}\left[\bar{h} \mathbf{E}_{\gamma, 1,-\gamma /(1-\gamma), A-}^{1} \partial_{5} \underline{L}(Z)\right]\right|_{t=0} ^{t=A}=0,
\end{aligned}
$$


where

$$
\begin{gathered}
Z=\left(t, \underline{x}^{*}(t, \alpha), \bar{x}^{*}(t, \alpha),{ }_{\mathrm{gH}}^{\mathrm{ABC}} D_{i 0+}^{\gamma} \underline{x}^{*}(t, \alpha),\right. \\
\left.{ }_{\mathrm{gBC}}^{\mathrm{ABC}} D_{i 0+}^{\gamma} \bar{x}^{*}(t, \alpha)\right), \quad i \in\{1,2\} .
\end{gathered}
$$

According to the end conditions of $\underline{h}(A, \alpha)=\bar{h}(A, \alpha)=$ $\underline{h}(b, \alpha)=\bar{h}(b, \alpha)=0$, the above equation can be deduced as follows:

$$
\begin{aligned}
& \int_{0}^{A}\left\{\underline{h}\left[{ }^{\mathrm{ABR}} D_{b-}^{\gamma} \partial_{4} \underline{L}(Z)-{ }^{\mathrm{ABR}} D_{A-}^{\gamma} \partial_{4} \underline{L}(Z)\right]\right. \\
& \left.+\bar{h}\left[{ }^{\mathrm{ABR}} D_{b-}^{\gamma} \partial_{5} \underline{L}(Z)-{ }^{\mathrm{ABR}} D_{A-}^{\gamma} \partial_{5} \underline{L}(Z)\right]\right\} d t \\
& +\int_{A}^{b}\left\{\underline{h}\left[\partial_{2} \underline{L}(Z)+{ }^{\mathrm{ABR}} D_{b-}^{\gamma} \partial_{4} \underline{L}(Z)\right]\right. \\
& \left.+\bar{h}\left[\partial_{3} \underline{L}(Z)+{ }^{\mathrm{ABR}} D_{b-}^{\gamma} \partial_{5} \underline{L}(Z)\right]\right\} d t \\
& +\frac{B(\gamma)}{1-\gamma}\left[\underline { h } ( 0 , \alpha ) \left(\mathbf{E}_{\gamma, 1,-\gamma /(1-\gamma), A_{-}} \partial_{4} \underline{L}(Z)\right.\right. \\
& \left.-\mathbf{E}_{\gamma, 1,-\gamma /(1-\gamma),{ }^{1}-} \partial_{4} \underline{L}(Z)\right)+\bar{h}(0, \alpha) \\
& \left.\cdot\left(\mathbf{E}_{\gamma, 1,-\gamma /(1-\gamma), A-}^{1} \partial_{5} \underline{L}(Z)-\mathbf{E}_{\gamma, 1,-\gamma /(1-\gamma), b-}^{1} \partial_{5} \underline{L}(Z)\right)\right] \\
& =0 .
\end{aligned}
$$

Since $\underline{h}(t, \alpha), \bar{h}(t, \alpha), \underline{h}(0, \alpha)$, and $\bar{h}(0, \alpha)$ are arbitrary elsewhere, we get the three necessary conditions

$$
\begin{aligned}
& { }^{\mathrm{ABR}} D_{b-}^{\gamma} \partial_{4} \underline{L}(Z)-{ }^{\mathrm{ABR}} D_{A_{-}}^{\gamma} \partial_{4} \underline{L}(Z)=0, \\
& { }^{\mathrm{ABR}} D_{b-}^{\gamma} \partial_{5} \underline{L}(Z)-{ }^{\mathrm{ABR}} D_{A_{-}}^{\gamma} \partial_{5} \underline{L}(Z)=0,
\end{aligned}
$$

on $[0, A]$,

$$
\begin{aligned}
& \partial_{2} \underline{L}(Z)+{ }^{\mathrm{ABR}} D_{b-}^{\gamma} \partial_{4} \underline{L}(Z)=0, \\
& \partial_{3} \underline{L}(Z)+{ }^{\mathrm{ABR}} D_{b-}^{\gamma} \partial_{5} \underline{L}(Z)=0,
\end{aligned}
$$

on $[A, b]$, and

$$
\begin{aligned}
& \mathbf{E}_{\gamma, 1,-\gamma /(1-\gamma), A-}^{1} \partial_{4} \underline{L}(Z)-\mathbf{E}_{\gamma, 1,-\gamma /(1-\gamma), b-}^{1} \partial_{4} \underline{L}(Z)=0, \\
& \mathbf{E}_{\gamma, 1,-\gamma /(1-\gamma), A_{-}}^{1} \partial_{5} \underline{L}(Z)-\mathbf{E}_{\gamma, 1,-\gamma /(1-\gamma), b-}^{1} \partial_{5} \underline{L}(Z)=0,
\end{aligned}
$$

at $t=0$.

Following the scheme above and computing $\left.\bar{j}^{\prime}(\epsilon)\right|_{\epsilon=0}=0$, we can also obtain

$$
\begin{aligned}
& { }^{\mathrm{ABR}} D_{b-}^{\gamma} \partial_{4} \bar{L}(Z)-{ }^{\mathrm{ABR}} D_{A_{-}}^{\gamma} \partial_{4} \bar{L}(Z)=0, \\
& { }^{\mathrm{ABR}} D_{b-}^{\gamma} \partial_{5} \bar{L}(Z)-{ }^{\mathrm{ABR}} D_{A_{-}}^{\gamma} \partial_{5} \bar{L}(Z)=0,
\end{aligned}
$$

on $[0, A]$,

$$
\begin{aligned}
& \partial_{2} \bar{L}(Z)+{ }^{\mathrm{ABR}} D_{b-}^{\gamma} \partial_{4} \bar{L}(Z)=0, \\
& \partial_{3} \bar{L}(Z)+{ }^{\mathrm{ABR}} D_{b-}^{\gamma} \partial_{5} \bar{L}(Z)=0,
\end{aligned}
$$

on $[A, b]$, and

$$
\begin{aligned}
& \mathbf{E}_{\gamma, 1,-\gamma /(1-\gamma), A-}^{1} \partial_{4} \bar{L}(Z)-\mathbf{E}_{\gamma, 1,-\gamma /(1-\gamma), b-}^{1} \partial_{4} \bar{L}(Z)=0, \\
& \mathbf{E}_{\gamma, 1,-\gamma /(1-\gamma), A-}^{1} \partial_{5} \bar{L}(Z)-\mathbf{E}_{\gamma, 1,-\gamma /(1-\gamma), b-}^{1} \partial_{5} \bar{L}(Z)=0,
\end{aligned}
$$

at $t=0$

Remark 32. The Euler-Lagrange equations (52) are easily extended to the case of several variations.

\section{The Weakly Sufficient \\ Optimality Conditions for Fuzzy Fractional Variational Problems}

In this section, we introduce the weak optimality conditions for fuzzy fractional variational problems. Let us consider the following multiobjective fractional variational problems defined for each $\alpha \in[0,1]$ :

$$
\begin{aligned}
\min & \left(\underline{J}^{\alpha}(\tilde{x}), \bar{J}^{\alpha}(\tilde{x})\right) \\
\text { where } & \underline{J}^{\alpha}(\tilde{x}) \\
& =\int_{0}^{b} \underline{L}\left(t, \tilde{x}(t),{ }_{\mathrm{gH}}^{\mathrm{ABC}} D_{i 0+}^{\gamma} \tilde{x}(t)\right) d t, \quad\left(\mathrm{MFVP}_{\alpha}\right) \\
& \bar{J}^{\alpha}(\tilde{x}) \\
& =\int_{0}^{b} \bar{L}\left(t, \tilde{x}(t),{ }_{\mathrm{gH}}^{\mathrm{ABC}} D_{i 0+}^{\gamma} \tilde{x}(t)\right) d t
\end{aligned}
$$

with $\widetilde{x}(t) \in C^{1}\left([0, b], \mathscr{R}_{f}\right) \cap L\left([0, b], \mathscr{R}_{f}\right), \widetilde{x}(0)=\widetilde{x}_{0}, \widetilde{x}(b)=$ $\tilde{x}_{b}$, and $i=1,2$, where $\tilde{x}_{0}, \tilde{x}_{b} \in \mathscr{R}_{f}$. The assumptions are the same as the problem of (FFVP).

Lemma 33. If $\widetilde{x}^{*}$ is a Pareto efficient solution for $\left(\mathrm{MFVP}_{\alpha}\right)$, for each $\alpha \in[0,1]$, then $\tilde{x}^{*}$ is a solution for (FFVP).

Proof. Suppose that $\tilde{x}^{*}$ is not a solution of (FFVP); then, there exists $\widetilde{\tilde{x}} \in \widetilde{N}_{\epsilon}\left(\widetilde{x}^{*}\right)$. From Definition 28, we get $\widetilde{\tilde{x}} \epsilon$ $C^{1}\left([0, b], \mathscr{R}_{f}\right) \cap L\left([0, b], \mathscr{R}_{f}\right)$ such that $\widetilde{J}(\widetilde{\tilde{x}}) \preceq \widetilde{J}\left(\widetilde{x}^{*}\right)$. In particular, there exists $\alpha^{*}$ such that

$$
\begin{aligned}
& \underline{J}^{\alpha^{*}}(\widetilde{\widetilde{x}}) \leq \underline{J}^{\alpha^{*}}\left(\tilde{x}^{*}\right), \\
& \bar{J}^{\alpha^{*}}(\widetilde{\widetilde{x}}) \leq \bar{J}^{\alpha^{*}}\left(\widetilde{x}^{*}\right)
\end{aligned}
$$

with a strict inequality, which contradicts the fact that $\tilde{x}^{*}$ is a Pareto efficient solution for $\left(\mathrm{MFVP}_{\alpha}\right)$.

Consider a weighting scalar problem for $\left(\mathrm{MFVP}_{\alpha}\right)$ as follows:

$$
\begin{aligned}
\min & I^{\alpha}(\tilde{x})+\bar{J}^{\alpha}(\tilde{x}) \\
\text { where } & I^{\alpha}(\tilde{x})=\int_{0}^{b} \underline{L}\left(t, \tilde{x}(t),{ }_{\mathrm{gH}}^{\mathrm{ABC}} D_{i 0+}^{\gamma} \tilde{x}(t)\right) d t, \quad\left(\mathrm{WSP}_{\alpha}\right) \\
& \bar{J}^{\alpha}(\tilde{x})=\int_{0}^{b} \bar{L}\left(t, \tilde{x}(t),{ }_{\mathrm{gH}}^{\mathrm{ABC}} D_{i 0+}^{\gamma} \tilde{x}(t)\right) d t
\end{aligned}
$$


with $\widetilde{x}(t) \in C^{1}\left([0, b], \mathscr{R}_{f}\right) \cap L\left([0, b], \mathscr{R}_{f}\right), \widetilde{x}(0)=\tilde{x}_{0}, \widetilde{x}(b)=$ $\tilde{x}_{b}$, and $i=1,2$, where $\tilde{x}_{0}, \tilde{x}_{b} \in \mathscr{R}_{f}$. The assumptions are the same as the problem of (FFVP).

Lemma 34. If $\tilde{x}^{*}$ is a local optimal solution of $\left(\mathrm{WSP}_{\alpha}\right)$, for each $\alpha \in[0,1]$, then $\tilde{x}^{*}$ is a solution of (FFVP).

Proof. Since $\left(\mathrm{WSP}_{\alpha}\right)$ is a weighting scalar problem for $\left(\mathrm{MFVP}_{\alpha}\right)$ and the weights are strictly positive, then $\tilde{x}^{*}$ is a Pareto efficient solution for $\left(\mathrm{MFVP}_{\alpha}\right)$ for all $\alpha \in[0,1]$. From Lemma $33, \widetilde{x}^{*}$ is a solution for (FFVP).

For the real-valued fractional variational problem $\left(\mathrm{WSP}_{\alpha}\right)$, we have the following necessary optimality condition.

Theorem 35. Let $\tilde{x}^{*}$ be a local optimal solution of $\left(\mathrm{WSP}_{\alpha}\right)$. Then, $\widetilde{x}^{*}$ is a solution of the fractional Euler-Lagrange equations

$$
\begin{aligned}
& \partial_{2}(\underline{L}+\bar{L})(Z)+{ }^{\mathrm{ABR}} D_{b-}^{\gamma} \partial_{4}(\underline{L}+\bar{L})(Z)=0, \\
& \partial_{3}(\underline{L}+\bar{L})(Z)+{ }^{\mathrm{ABR}} D_{b-}^{\gamma} \partial_{5}(\underline{L}+\bar{L})(Z)=0,
\end{aligned}
$$

for all $t \in[0, b]$, where

$$
\begin{gathered}
Z=\left(t, \underline{x}^{*}(t, \alpha), \bar{x}^{*}(t, \alpha),{ }_{g H}^{\mathrm{ABC}} D_{i 0+}^{\gamma} \underline{x}^{*}(t, \alpha),\right. \\
\left.{ }_{g H}^{\mathrm{ABC}} D_{i 0+}^{\gamma} \bar{x}^{*}(t, \alpha)\right), \quad i \in\{1,2\} .
\end{gathered}
$$

Proof. Let $\widetilde{x}^{*}(t)=\left[\underline{x}^{*}(t, \alpha), \bar{x}^{*}(t, \alpha)\right] \in \widetilde{X}_{0 d}$ be a solution of $\left(\mathrm{WSP}_{\alpha}\right)$ and $\widetilde{h}(t)=[\underline{h}(t, \alpha), \bar{h}(t, \alpha)]:[0, b] \rightarrow \mathscr{R}_{f}$ be $[(i)-\mathrm{gH}]_{\gamma}^{\mathrm{ABC}}$-differentiable fuzzy function with the end condition of $\underline{h}(0, \alpha)=\bar{h}(0, \alpha)=\underline{h}(b, \alpha)=\bar{h}(b, \alpha)=0$ for $i=1,2$ and $\alpha, \gamma \in[0,1]$. For $|\epsilon| \ll 1$, we have $\widetilde{x}^{*}+\epsilon \widetilde{h} \in \widetilde{N}_{\epsilon}\left(\widetilde{x}^{*}\right)$.

Let $j$ be the function defined in a neighborhood of zero as

$$
j(\epsilon)=\underline{J}\left(\tilde{x}^{*}+\epsilon \widetilde{h}\right)+\bar{J}\left(\widetilde{x}^{*}+\epsilon \widetilde{h}\right) .
$$

Since the function $\tilde{x}^{*}(t)$ is a local optimal solution of (WSP ${ }_{\alpha}$ ), then $\epsilon=0$ is a minimizer of $j(\epsilon)$. Therefore, we get

$$
\left.\frac{d j(\epsilon)}{d \epsilon}\right|_{\epsilon=0}=0 .
$$

Computing $\left.(d j(\epsilon) / d \epsilon)\right|_{\epsilon=0}$, we obtain

$$
\begin{aligned}
& \left.\frac{d j(\epsilon)}{d \epsilon}\right|_{\epsilon=0}=\left.\frac{d\left[\underline{J}^{\alpha}\left(\tilde{x}^{*}+\epsilon \widetilde{h}\right)+\bar{J}^{\alpha}\left(\tilde{x}^{*}+\epsilon \widetilde{h}\right)\right]}{d \epsilon}\right|_{\epsilon=0} \\
& =\int_{0}^{b} \frac{d}{d \epsilon}[(\underline{L}+\bar{L}) \\
& \left.\cdot\left(t, \tilde{x}^{*}(t)+\epsilon \widetilde{h}(t),{ }_{\mathrm{gH}}^{\mathrm{ABC}} D_{i 0+}^{\gamma}\left(\widetilde{x}^{*}(t)+\epsilon \widetilde{h}(t)\right)\right)\right]\left.\right|_{\epsilon=0} d t
\end{aligned}
$$

$$
\begin{aligned}
& =\left.\int_{0}^{b} \frac{d}{d \epsilon}[(\underline{L}+\bar{L})(\diamond)]\right|_{\epsilon=0} d t=\int_{0}^{b}\left[\partial_{2}(\underline{L}+\bar{L})(Z)\right. \\
& \cdot \underline{h}(t, \alpha)+\partial_{3}(\underline{L}+\bar{L})(Z) \bar{h}(t, \alpha)+\partial_{4}(\underline{L}+\bar{L})(Z) \\
& \cdot{ }^{\mathrm{ABC}} D_{0+}^{\gamma}(\underline{h}(t, \alpha))+\partial_{5}(\underline{L}+\bar{L})(Z) \\
& \left.\cdot{ }^{\mathrm{ABC}} D_{0+}^{\gamma}(\bar{h}(t, \alpha))\right] d t,
\end{aligned}
$$

where

$$
\begin{aligned}
& (\diamond)=\left(t, \underline{x}^{*}(t, \alpha)+\epsilon \underline{h}(t, \alpha), \bar{x}^{*}(t, \alpha)+\epsilon \bar{h}(t, \alpha),\right. \\
& { }_{\mathrm{gH}}^{\mathrm{ABC}} D_{i 0+}^{\gamma} \underline{x}^{*}(t, \alpha)+\epsilon^{\mathrm{ABC}} D_{0+}^{\gamma}(\underline{h}(t, \alpha)), \\
& \left.{ }_{\mathrm{gB}}^{\mathrm{ABC}} D_{i 0+}^{\gamma} \bar{x}^{*}(t, \alpha)+\epsilon^{\mathrm{ABC}} D_{0+}^{\gamma} \bar{h}(t, \alpha)\right), \\
& (Z)=\left(t, \underline{x}^{*}(t, \alpha), \bar{x}^{*}(t, \alpha),{ }_{\mathrm{gH}}^{\mathrm{ABC}} D_{i 0+}^{\gamma} \underline{x}^{*}(t, \alpha),\right. \\
& \left.{ }_{\mathrm{gB}}^{\mathrm{ABC}} D_{i 0+}^{\gamma} \bar{x}^{*}(t, \alpha)\right),
\end{aligned}
$$

$$
i=1,2 \text {. }
$$

Using Proposition 18, we have

$$
\begin{aligned}
& \int_{0}^{b} \partial_{4}(\underline{L}+\bar{L})(Z)^{\mathrm{ABC}} D_{0+}^{\gamma} \underline{h} d t \\
& =\int_{0}^{b} \underline{h}^{\mathrm{ABR}} D_{b-}^{\gamma} \partial_{4}(\underline{L}+\bar{L})(Z) d t \\
& \quad+\left.\frac{B(\gamma)}{1-\gamma}\left[\underline{h} \mathbf{E}_{\gamma, 1,-\gamma /(1-\gamma), b-}^{1} \partial_{4}(\underline{L}+\bar{L})(Z)\right]\right|_{t=0} ^{t=b}, \\
& \int_{0}^{b} \partial_{5}(\underline{L}+\bar{L})(Z)^{\mathrm{ABC}} D_{0+}^{\gamma} \bar{h} d t \\
& =\int_{0}^{b} \bar{h}^{\mathrm{ABR}} D_{b-}^{\gamma} \partial_{5}(\underline{L}+\bar{L})(Z) d t \\
& \quad+\left.\frac{B(\gamma)}{1-\gamma}\left[\bar{h} \mathbf{E}_{\gamma, 1,-\gamma /(1-\gamma), b-}^{1} \partial_{5}(\underline{L}+\bar{L})(Z)\right]\right|_{t=0} ^{t=b} .
\end{aligned}
$$

So, we get

$$
\begin{aligned}
& \left.\frac{d j(\epsilon)}{d \epsilon}\right|_{\epsilon=0} \\
& =\int_{0}^{b}\left\{\left[\partial_{2}(\underline{L}+\bar{L})(Z)+{ }^{\mathrm{ABR}} D_{b_{-}}^{\gamma} \partial_{4}(\underline{L}+\bar{L})(Z)\right]\right. \\
& \cdot \underline{h}(t, \alpha) \\
& +\left[\partial_{3}(\underline{L}+\bar{L})(Z)+{ }^{\mathrm{ABR}} D_{b-}^{\gamma} \partial_{5}(\underline{L}+\bar{L})(Z)\right] \\
& \cdot \bar{h}(t, \alpha)\} d t=0 .
\end{aligned}
$$

Since $\widetilde{h}(t)=[\underline{h}(t, \alpha), \bar{h}(t, \alpha)] \in \widetilde{X}_{0 d}$ is arbitrary elsewhere, we have the fractional Euler-Lagrange equations (84). 
Definition 36. One says that the real-valued function $L(t$, $x, y, z, q)$ is convex in $K \subseteq \mathbf{R}^{5}$ if $\partial_{j} L$ exist and are continuous for $j=1,2,3,4,5$, and the condition

$$
\begin{gathered}
L\left(t, x+x_{1}, y+y_{1}, z+z_{1}, q+q_{1}\right)-L(t, x, y, z, q) \\
\geq \partial_{2} L(t, x, y, z, q) x_{1}+\partial_{3} L(t, x, y, z, q) y_{1} \\
\quad+\partial_{4} L(t, x, y, z, q) z_{1}+\partial_{5} L(t, x, y, z, q) q_{1}
\end{gathered}
$$

holds for every $(t, x, y, z, q),\left(t, x+x_{1}, y+y_{1}, z+z_{1}, q+q_{1}\right) \in K$.

Theorem 37. If function $\underline{L}+\bar{L}$ as in $\left(\mathrm{WSP}_{\alpha}\right)$ is convex in $[a, b] \times \mathbf{R}^{5}$, then each solution of the fractional Euler-Lagrange equations (84) minimizes (FFVP), when restricted to the boundary conditions of $\widetilde{x}(0)=\widetilde{x}_{0}$ and $\widetilde{x}(b)=\widetilde{x}_{b}$.

Proof. Let $\widetilde{x}(t)=[\underline{x}(t, \alpha), \bar{x}(t, \alpha)] \in \widetilde{X}_{0 d}$ be a solution of $(84) ; \widetilde{h}(t)=[\underline{h}(t, \alpha), \bar{h}(t, \alpha)]:[0, b] \rightarrow \mathscr{R}_{f}$ is $[(i)-\mathrm{gH}]_{\gamma}^{\mathrm{ABC}}-$ differentiable fuzzy function with the end condition of $\underline{h}(0$, $\alpha)=\bar{h}(0, \alpha)=\underline{h}(b, \alpha)=\bar{h}(b, \alpha)=0$ for $i=1,2$ and $\alpha$, $\gamma \in[0,1]$. For $|\epsilon| \ll 1$, we have $\widetilde{x}+\epsilon \widetilde{h} \in \widetilde{N}_{\epsilon}(\widetilde{x})$.

Since the function $\underline{L}+\bar{L}$ as in $\left(\mathrm{WSP}_{\alpha}\right)$ is convex, we get

$$
\begin{aligned}
& {\left[\underline{J}^{\alpha}(\tilde{x}+\epsilon \widetilde{h})+\bar{J}^{\alpha}(\tilde{x}+\epsilon \widetilde{h})\right]-\left[\underline{J}^{\alpha}(\widetilde{x})+\bar{J}^{\alpha}(\tilde{x})\right]} \\
& \quad=\int_{0}^{b}[(\underline{L}+\bar{L}) \\
& \cdot\left(t, \tilde{x}(t)+\epsilon \widetilde{h}(t),{ }_{\mathrm{gH}}^{\mathrm{ABC}} D_{i 0+}^{\gamma}(\tilde{x}(t)+\epsilon \widetilde{h}(t))\right) \\
& \left.\quad-(\underline{L}+\bar{L})\left(t, \tilde{x}(t),{ }_{\mathrm{gH}}^{\mathrm{ABC}} D_{i 0+}^{\gamma}(\widetilde{x}(t))\right)\right] d t \\
& \quad=\int_{0}^{b}[(\underline{L}+\bar{L})(\diamond)-(\underline{L}+\bar{L})(Z)] d t \\
& \quad \geq \int_{0}^{b}\left[\partial_{2}(\underline{L}+\bar{L})(Z) \epsilon \underline{h}(t, \alpha)+\partial_{3}(\underline{L}+\bar{L})(Z)\right. \\
& \cdot \epsilon \bar{h}(t, \alpha)+\partial_{4}(\underline{L}+\bar{L})(Z) \epsilon^{\mathrm{ABC}} D_{0+}^{\gamma}(\underline{h}(t, \alpha)) \\
& \left.+\partial_{5}(\underline{L}+\bar{L})(Z) \epsilon^{\mathrm{ABC}} D_{0+}^{\gamma}(\bar{h}(t, \alpha))\right] d t,
\end{aligned}
$$

where

$$
\begin{aligned}
& (\diamond)=\left(t, \underline{x}^{*}(t, \alpha)+\epsilon \underline{h}(t, \alpha), \bar{x}^{*}(t, \alpha)+\epsilon \bar{h}(t, \alpha),\right. \\
& { }_{\mathrm{gH}}^{\mathrm{ABC}} D_{i 0+}^{\gamma} \underline{x}^{*}(t, \alpha)+\epsilon^{\mathrm{ABC}} D_{0+}^{\gamma}(\underline{h}(t, \alpha)), \\
& \left.{ }_{\mathrm{gB}}^{\mathrm{ABC}} D_{i 0+}^{\gamma} \bar{x}^{*}(t, \alpha)+\epsilon^{\mathrm{ABC}} D_{0+}^{\gamma} \bar{h}(t, \alpha)\right), \\
& (Z)=\left(t, \underline{x}^{*}(t, \alpha), \bar{x}^{*}(t, \alpha),{ }_{\mathrm{gH}}^{\mathrm{ABC}} D_{i 0+}^{\gamma} \underline{x}^{*}(t, \alpha),\right. \\
& \left.\underset{\mathrm{gH}}{\mathrm{ABC}} D_{i 0+}^{\gamma} \bar{x}^{*}(t, \alpha)\right),
\end{aligned}
$$

Using Proposition 18, we can obtain (90). According to the end condition of $\underline{h}(0, \alpha)=\bar{h}(0, \alpha)=\underline{h}(b, \alpha)=\bar{h}(b, \alpha)=0$ and (90), we have

$$
\begin{aligned}
& {\left[\underline{J}^{\alpha}(\tilde{x}+\epsilon \widetilde{h})+\bar{J}^{\alpha}(\tilde{x}+\epsilon \widetilde{h})\right]-\left[\underline{J}^{\alpha}(\tilde{x})+\bar{J}^{\alpha}(\tilde{x})\right]} \\
& \quad \geq \int_{0}^{b}\left\{\left[\partial_{2}(\underline{L}+\bar{L})(Z)+{ }^{\mathrm{ABR}} D_{b-}^{\gamma} \partial_{4}(\underline{L}+\bar{L})(Z)\right]\right. \\
& \cdot \epsilon \underline{h}(t, \alpha)+\left[\partial_{3}(\underline{L}+\bar{L})(Z)\right. \\
& \left.\left.\quad+{ }^{\mathrm{ABR}} D_{b-}^{\gamma} \partial_{5}(\underline{L}+\bar{L})(Z)\right] \epsilon \bar{h}(t, \alpha)\right\} d t .
\end{aligned}
$$

Since $\widetilde{x}(t)$ is a solution of (84), then

$$
\left[I^{\alpha}(\widetilde{x}+\epsilon \widetilde{h})+\bar{J}^{\alpha}(\tilde{x}+\epsilon \widetilde{h})\right] \geq\left[I^{\alpha}(\widetilde{x})+\bar{J}^{\alpha}(\widetilde{x})\right],
$$

which means that $\widetilde{x}(t)$ is a local optimal solution of $\left(\mathrm{WSP}_{\alpha}\right)$. From Lemma 34, $\widetilde{x}(t)$ is also a solution of (FFVP).

\section{Example}

Example 1. We consider the following (FFVP) to find the minimum of

$$
\begin{aligned}
\min \tilde{J}(\tilde{x})=\int_{0}^{1}\left({ }_{\mathrm{gH}}^{\mathrm{ABC}} D_{i 0+}^{\gamma} \tilde{x}(t)\right)^{2} d t, \\
i=1,2,0<\alpha, \quad \gamma<1
\end{aligned}
$$

subject to $\tilde{x}(0)=0$,

$$
\widetilde{x}(1)=[2+\alpha, 4-\alpha] \cdot \frac{\gamma}{B(\gamma) \Gamma(\gamma)} .
$$

We first derive the $\alpha$-level set of $\widetilde{J}$ as follows:

$$
\begin{aligned}
& {[\widetilde{J}(\widetilde{x})]^{\alpha}} \\
& =\int_{0}^{1}\left(\underline{\left({ }_{\mathrm{gH}}^{\mathrm{ABC}} D_{i 0+}^{\gamma} \tilde{x}(t)\right)^{2}}, \overline{\left({ }_{\mathrm{gH}}^{\mathrm{ABC}} D_{i 0+}^{\gamma} \tilde{x}(t)\right)^{2}}\right) d t, \\
& i=1,2 .
\end{aligned}
$$

According to Definition 23, if $i=1$, then

$$
\left[{ }_{\mathrm{gH}}^{\mathrm{ABC}} D_{i 0+}^{\gamma} \tilde{x}(t)\right]^{\alpha}=\left[{ }^{\mathrm{ABC}} D_{0+}^{\gamma} \underline{x}(t, \alpha),{ }^{\mathrm{ABC}} D_{0+}^{\gamma} \bar{x}(t, \alpha)\right],
$$

and if $i=2$, then

$$
\begin{aligned}
& {\left[{ }_{\mathrm{gH}}^{\mathrm{ABC}} D_{i 0+}^{\gamma} \widetilde{x}(t)\right]^{\alpha}} \\
& \quad=\left[{ }^{\mathrm{ABC}} D_{0+}^{\gamma} \bar{x}(t, \alpha),{ }^{\mathrm{ABC}} D_{0+}^{\gamma} \underline{x}(t, \alpha)\right] .
\end{aligned}
$$

From Theorem 30, the Euler-Lagrange equations are given as

$$
\begin{aligned}
{ }^{\mathrm{ABR}} D_{1-}^{\gamma}\left({ }^{\mathrm{ABC}} D_{0+}^{\gamma} \underline{x}(t, \alpha)\right) & =0, \\
{ }^{\mathrm{ABR}} D_{1-}^{\gamma}\left({ }^{\mathrm{ABC}} D_{0+}^{\gamma} \gamma \bar{x}(t, \alpha)\right) & =0,
\end{aligned}
$$


with $\tilde{x}(0)=0, \tilde{x}(1)=[2+\alpha, 4-\alpha] \cdot(\gamma / B(\gamma) \Gamma(\gamma))$ for $0<$ $\alpha, \gamma<1$. have

According to Theorem 2.1 and Proposition 3.4 in [20], we

$$
\begin{aligned}
& { }^{\mathrm{AB}} I_{1-}^{\gamma}\left({ }^{\mathrm{ABR}} D_{1-}^{\gamma} f(t)\right)=f(t), \\
& { }^{\mathrm{AB}} I_{0+}^{\gamma}\left({ }^{\mathrm{ABC}} D_{0+}^{\gamma} f(t)\right)=f(t)-f(0) .
\end{aligned}
$$

For (101), by applying ${ }^{\mathrm{AB}} I_{1-}^{\gamma}$ to both sides, we obtain

$$
\begin{aligned}
& { }^{\mathrm{ABC}} D_{0+}^{\gamma} \underline{x}(t, \alpha)=0, \\
& { }^{\mathrm{ABC}} D_{0+}^{\gamma} \bar{x}(t, \alpha)=0 .
\end{aligned}
$$

By applying ${ }^{\mathrm{AB}} I_{0+}^{\gamma}$ to both sides of (103), we get

$$
\begin{aligned}
& \underline{x}(t, \alpha)=\underline{x}(0, \alpha)+k_{1} \cdot \frac{\gamma t^{\gamma-1}}{B(\gamma) \Gamma(\gamma)}, \\
& \bar{x}(t, \alpha)=\bar{x}(0, \alpha)+k_{2} \cdot \frac{\gamma t^{\gamma-1}}{B(\gamma) \Gamma(\gamma)},
\end{aligned}
$$

since, according to Remark 3.1 in [20], the nonzero function $g(t)=\gamma t^{\gamma-1} / B(\gamma) \Gamma(\gamma)$ whose fractional $\mathrm{ABC}$ derivative is zero. have

From $\tilde{x}(0)=0, \tilde{x}(1)=[2+\alpha, 4-\alpha] \cdot(\gamma / B(\gamma) \Gamma(\gamma))$, we

$$
\begin{aligned}
& \underline{x}(t, \alpha)=(2+\alpha) \cdot \frac{\gamma t^{\gamma-1}}{B(\gamma) \Gamma(\gamma)}, \\
& \bar{x}(t, \alpha)=(4-\alpha) \cdot \frac{\gamma t^{\gamma-1}}{B(\gamma) \Gamma(\gamma)}
\end{aligned}
$$

So, the solution is

$$
[\tilde{x}(t)]^{\alpha}=[2+\alpha, 4-\alpha] \cdot \frac{\gamma t^{\gamma-1}}{B(\gamma) \Gamma(\gamma)}
$$

for $0<\alpha, \gamma<1$ and $t \in[0,1]$.

\section{Conclusions}

In this paper, We studied the $\mathrm{AB}$ fractional derivative of fuzzy functions based on the generalized Hukuhara difference. Under the condition of $\mathrm{gH}-\mathrm{AB}$ fractional differentiability, we proposed the necessary and sufficient optimality conditions for problems of the fractional calculus of variations with a Lagrange function depending on the $\mathrm{AB}$ fractional derivative. The advantage of the new fractional derivative has no singularity and no locality. However, the Euler-Lagrange equations of (FFVP) are very difficult to solve; we should develop numerical methods to solve this problem. As future works, we should also study which type of the fractional order derivative is more appropriate in the actual variational problem.

\section{Conflicts of Interest}

The authors declare that they have no conflicts of interest.

\section{Acknowledgments}

This work is supported by the National Natural Science Foundation of China (Grants nos. 11701446, 11601420, 11401469, and 60974082) and the Science Plan Foundation of the Education Bureau of Shaanxi Province (no. 2013JK 1130).

\section{References}

[1] F. Riewe, "Nonconservative Lagrangian and Hamiltonian mechanics," Physical Review E: Statistical, Nonlinear, and Soft Matter Physics, vol. 53, no. 2, pp. 1890-1899, 1996.

[2] O. P. Agrawal, "Formulation of Euler-Lagrange equations for fractional variational problems," Journal of Mathematical Analysis and Applications, vol. 272, no. 1, pp. 368-379, 2002.

[3] O. P. Agrawal, "Fractional variational calculus and the transversality conditions," Journal of Physics A: Mathematical and General, vol. 39, no. 33, pp. 10375-10384, 2006.

[4] O. P. Agrawal, "Fractional variational calculus in terms of Riesz fractional derivatives," Journal of Physics A: Mathematical and General, vol. 40, no. 24, pp. 6287-6303, 2007.

[5] R. Almeida, "Fractional variational problems with the RieszCaputo derivative," Applied Mathematics Letters, vol. 25, no. 2, pp. 142-148, 2012

[6] R. Almeida, "Variational problems involving a Caputo-type fractional derivative," Journal of Optimization Theory and Applications, vol. 174, no. 1, pp. 276-294, 2017.

[7] R. Almeida, "Fractional variational problems depending on indefinite integrals and with delay," Bulletin of the Malaysian Mathematical Sciences Society, vol. 39, no. 4, pp. 1515-1528, 2016.

[8] Y. Xu and O. P. Agrawal, "New fractional operators and application to fractional variational problem," Computers of Mathematics with Applications, 2016.

[9] J. Zhang, X. Ma, and L. Li, “Optimality conditions for fractional variational problems with Caputo-Fabrizio fractional derivatives," Advances in Difference Equations, Paper No. 357, 14 pages, 2017.

[10] M. Caputo and M. Fabrizio, "A new definition of fractional derivative without singular kernel," Progress in Fractional Differentiation and Applications, vol. 1, no. 2, pp. 73-85, 2015.

[11] J. Lozada and J. J. Nieto, "Properties of a new fractional derivative without singular kernel," Progress in Fractional Differentiation and Applications, vol. 1, no. 2, pp. 87-92, 2015.

[12] D. Baleanu, A. Mousalou, and S. Rezapour, "A new method for investigating approximate solutions of some fractional integrodifferential equations involving the Caputo-Fabrizio derivative," Advances in Difference Equations, vol. 2017, no. 1, article no. 51, 2017.

[13] N. A. Sheikh, F. Ali, M. Saqib et al., "Comparison and analysis of the Atangana-Baleanu and Caputo-Fabrizio fractional derivatives for generalized Casson fluid model with heat generation and chemical reaction," Results in Physics, vol. 7, pp. 789-800, 2017.

[14] N. A. Sheikh, F. Ali, M. Saqib, I. Khan, and S. A. A. Jan, "A comparative study of Atangana-Baleanu and Caputo-Fabrizio fractional derivatives to the convective flow of a generalized Casson fluid," The European Physical Journal Plus, vol. 132, no. 1, article no. 54, 2017.

[15] A. Atangana and I. Koca, "Chaos in a simple nonlinear system with Atangana-Baleanu derivatives with fractional order," Chaos, Solitons \& Fractals, vol. 89, pp. 447-454, 2016. 
[16] I. Koca and A. Atangana, "Solutions of Cattaneo-Hristov model of elastic heat diffusion with Caputo-Fabrizio and AtanganaBaleanu fractional derivatives," THERMAL SCIENCE, vol. 2016, 2016.

[17] X. J. Yang, F. Gao, and J. A. Machado, A New Fractional Derivative Involving The Normalized Sinc Function without Singular Kernel, 2017.

[18] A. Atangana and D. Baleanu, "New fractional derivatives with nonlocal and non-singular kernel: theory and application to heat transfer model," THERMAL SCIENCE, vol. 20, no. 2, pp. 763-769, 2016.

[19] O. J. Algahtani, "Comparing the Atangana-Baleanu and Caputo-Fabrizio derivative with fractional order: ALLen CAHn model," Chaos, Solitons \& Fractals, vol. 89, pp. 552-559, 2016.

[20] T. Abdeljawad and D. Baleanu, "Integration by parts and its applications of a new nonlocal fractional derivative with MittagLeffler nonsingular kernel," The Journal of Nonlinear Science and Applications, vol. 10, no. 03, pp. 1098-1107, 2017.

[21] B. Farhadinia, "Necessary optimality conditions for fuzzy variational problems," Information Sciences, vol. 181, no. 7, pp. 13481357, 2011

[22] O. S. Fard and M. Salehi, "A survey on fuzzy fractional variational problems," Journal of Computational and Applied Mathematics, vol. 271, pp. 71-82, 2014.

[23] J. Soolaki, O. S. Fard, and A. H. Borzabadi, "Generalized EulerLagrange equations for fuzzy fractional variational calculus," Mathematical Communications, vol. 21, no. 2, pp. 199-218, 2016.

[24] B. Bede and L. Stefanini, "Generalized differentiability of fuzzyvalued functions," Fuzzy Sets and Systems, vol. 230, pp. 119-141, 2013.

[25] Y. Chalco-Cano, W. A. Lodwick, R. Osuna-Gomez, and A. Rufian-Lizana, "The Karush-KUHn-Tucker optimality conditions for fuzzy optimization problems," Fuzzy Optimization and Decision Making. A Journal of Modeling and Computation Under Uncertainty, vol. 15, no. 1, pp. 57-73, 2016.

[26] B. van Brunt, The calculus of variations, Universitext, SpringerVerlag, New York, 2004.

[27] T. Allahviranloo, A. Armand, and Z. Gouyandeh, "Fuzzy fractional differential equations under generalized fuzzy Caputo derivative," Journal of Intelligent \& Fuzzy Systems: Applications in Engineering and Technology, vol. 26, no. 3, pp. 1481-1490, 2014. 


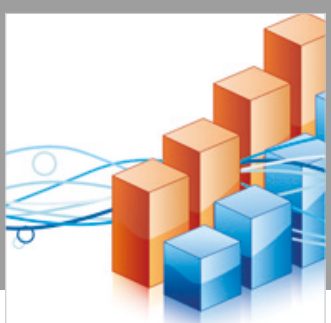

Advances in

Operations Research

\section{-n-m}
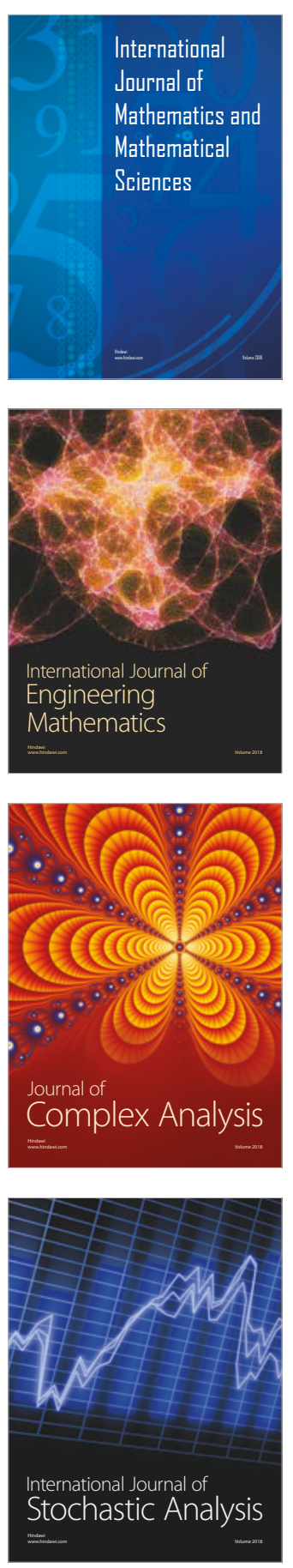
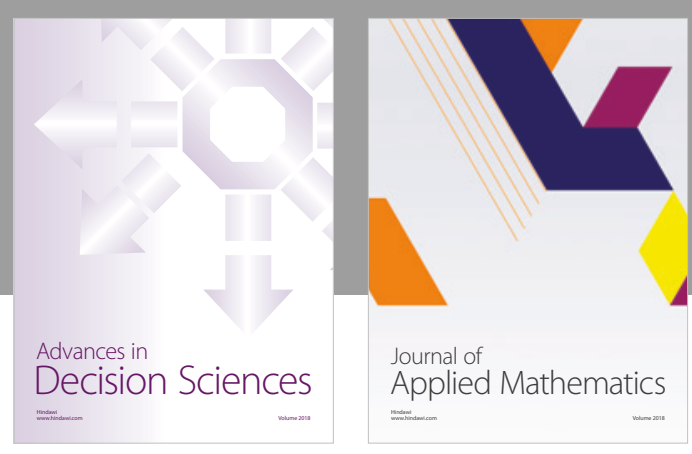

Journal of

Applied Mathematics
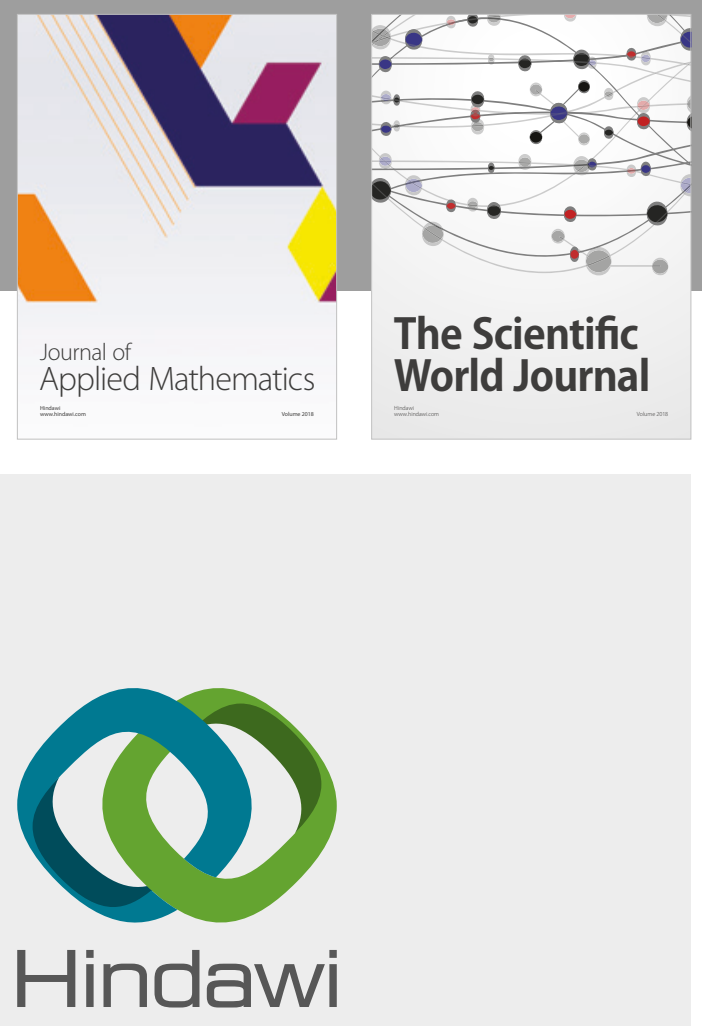

Submit your manuscripts at

www.hindawi.com

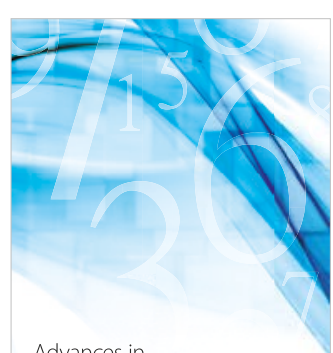

Advances in
Numerical Analysis
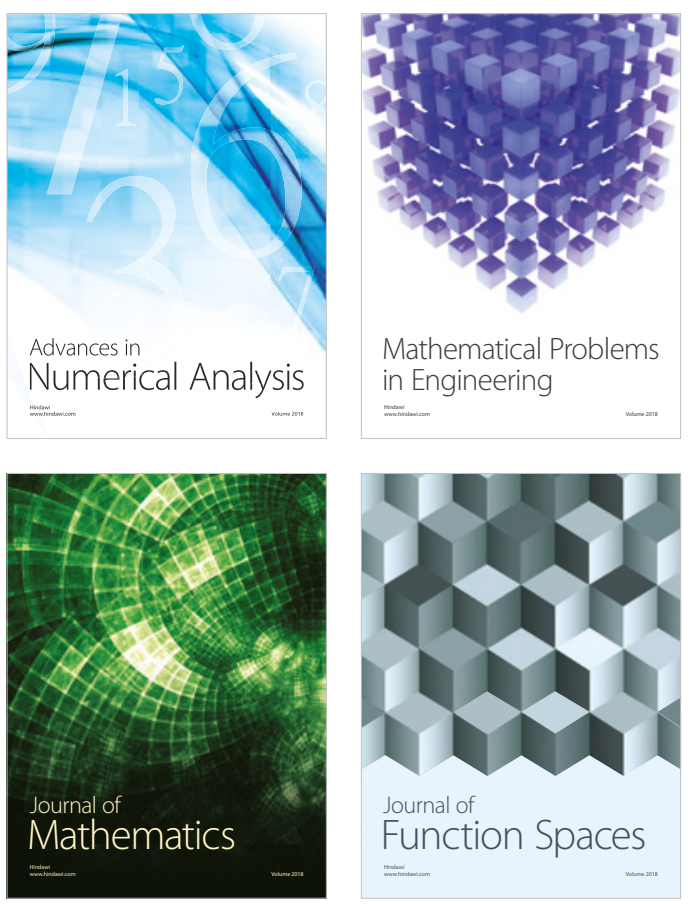

Mathematical Problems in Engineering

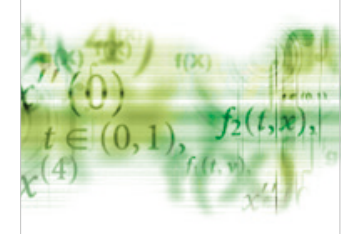

International Journal of

Differential Equations

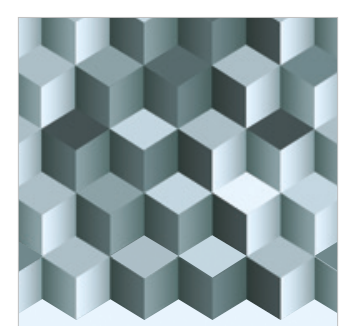

Journal of

Function Spaces

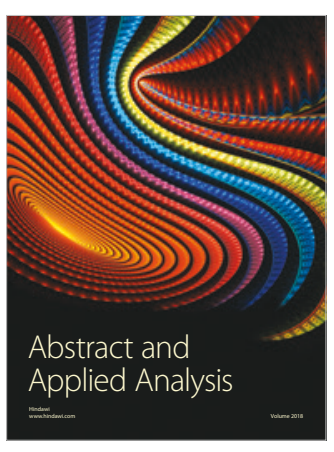

The Scientific

World Journal

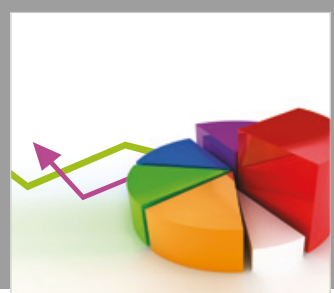

Journal of

Probability and Statistics
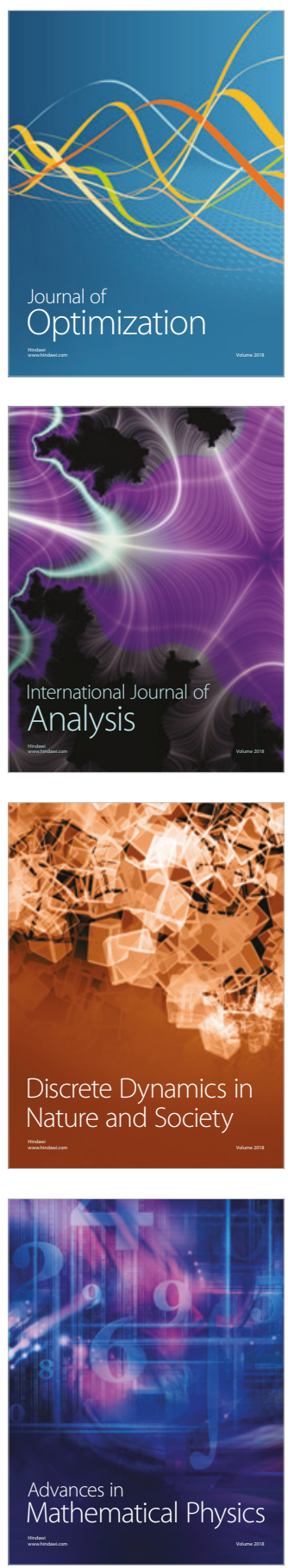